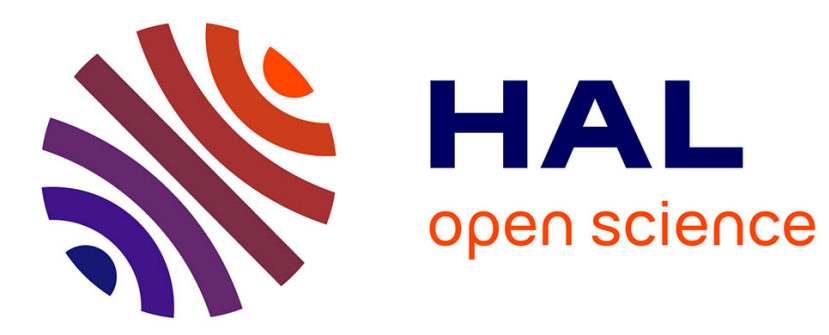

\title{
Stability of a Gaussian pancake vortex in a stratified fluid
}

\author{
M. Eletta Negretti, Paul Billant
}

\section{To cite this version:}

M. Eletta Negretti, Paul Billant. Stability of a Gaussian pancake vortex in a stratified fluid. Journal of Fluid Mechanics, 2013, 718, pp.457-480. 10.1017/jfm.2012.624 . hal-00996485

\section{HAL Id: hal-00996485}

https://hal-polytechnique.archives-ouvertes.fr/hal-00996485

Submitted on 2 Jun 2014

HAL is a multi-disciplinary open access archive for the deposit and dissemination of scientific research documents, whether they are published or not. The documents may come from teaching and research institutions in France or abroad, or from public or private research centers.
L'archive ouverte pluridisciplinaire HAL, est destinée au dépôt et à la diffusion de documents scientifiques de niveau recherche, publiés ou non, émanant des établissements d'enseignement et de recherche français ou étrangers, des laboratoires publics ou privés. 


\title{
Stability of a Gaussian pancake vortex in a stratified fluid
}

\author{
M. Eletta Negretti $\uparrow$ and Paul Billant \\ LadHyX, CNRS, Ecole Polytechnique, 91128 Palaiseau CEDEX, France \\ (Received 22 February 2012; revised 8 November 2012; accepted 12 December 2012; \\ first published online 8 February 2013)
}

Vortices in stably stratified fluids generally have a pancake shape with a small vertical thickness compared with their horizontal size. In order to understand what mechanism determines their minimum thickness, the linear stability of an axisymmetric pancake vortex is investigated as a function of its aspect ratio $\alpha$, the horizontal Froude number $F_{h}$, the Reynolds number $R e$ and the Schmidt number $S c$. The vertical vorticity profile of the base state is chosen to be Gaussian in both radial and vertical directions. The vortex is unstable when the aspect ratio is below a critical value, which scales with the Froude number: $\alpha_{c} \sim 1.1 F_{h}$ for sufficiently large Reynolds numbers. The most unstable perturbation has an azimuthal wavenumber either $m=0,|m|=1$ or $|m|=2$ depending on the control parameters. We show that the threshold corresponds to the appearance of gravitationally unstable regions in the vortex core due to the thermal wind balance. The Richardson criterion for shear instability based on the vertical shear is never satisfied alone. The dominance of the gravitational instability over the shear instability is shown to hold for a general class of pancake vortices with angular velocity of the form $\tilde{\Omega}(r, z)=\Omega(r) f(z)$ provided that $r \partial \Omega / \partial r<3 \Omega$ everywhere. Finally, the growth rate and azimuthal wavenumber selection of the gravitational instability are accounted well by considering an unstably stratified viscous and diffusive layer in solid body rotation with a parabolic density gradient.

Key words: buoyancy-driven instability, stratified turbulence, vortex instability

\section{Introduction}

Stably stratified flows are generally organized into layers with a small thickness compared with their horizontal extent (Riley \& Lelong 2000). Such layered structure can appear spontaneously on a vertically uniform flow through the zigzag instability (Billant \& Chomaz 2000a; Otheguy, Chomaz \& Billant 2006; Billant 2010; Billant et al. 2010). The typical vertical thickness of the layers is then $U / N$, where $U$ is the characteristic horizontal velocity and $N$ is the Brunt-Väisälä frequency. The zigzag instability is a mechanism which imposes an upper bound on the vertical scale of stratified flows, i.e. if vortices are too tall, they may experience the zigzag instability and break into pancake vortices with a smaller vertical size. In contrast, what determines the minimum thickness of the layers and pancake vortices is not well understood. Riley \& DeBruynKops (2003) and Brethouwer et al. (2007) reported 
in simulations of stratified turbulence the presence of shear instabilities which may appear when the vertical shear is too high, i.e. when the layers are too thin. Deloncle, Billant \& Chomaz (2008) also reported the onset of a shear instability due to the vertical shear generated by the development of the zigzag instability on a counterrotating vortex pair. In contrast, Waite \& Smolarkiewicz (2008) observed a gravitational instability on a similar flow whereas Augier \& Billant (2011) observed both shear and gravitational instabilities.

In order to better understand what mechanism determines the minimum thickness of the layers in stably stratified flows, we investigate here the stability of a single axisymmetric pancake vortex in a stably stratified fluid as a function of its aspect ratio.

The dynamics of a single pancake vortex in a stratified fluid has been studied experimentally by Flor \& van Heijst (1996). For strong stratification (i.e. low Froude number), the pancake vortices were observed to be stable and to decay by vertical diffusion. For weaker stratification, the vortices were unstable and evolved into multipolar vortices (see also Beckers et al. 2003). The instability was attributed to a barotropic shear instability since the radial profile of vorticity presented an extremum and is thus unstable according to the Rayleigh inflection point theorem.

The three-dimensional structure of stable pancake vortices has been investigated experimentally by Bonnier, Eiff \& Bonneton (2000) and Beckers et al. (2001). They have measured the pinching of the isopycnals which is due to the cyclostrophic and hydrostatic balances. Beckers et al. (2001) have studied analytically and numerically the viscous diffusion of a pancake vortex with a Gaussian angular velocity in both radial and vertical directions. They reported that for some combinations of aspect ratio and Froude number, the density gradient can be locally positive, i.e. gravitationally unstable. Beckers et al. (2001) and Godoy-Diana \& Chomaz (2003) have also found that, when momentum and buoyancy do not diffuse at the same rate, the viscous decay of the vortex induces a secondary three-dimensional circulation that modifies the velocity decay rate.

Fung (1986) derived three sufficient conditions for stability based on three Richardson numbers for a general class of rotating flows with the velocity and density perturbations varying in both the axial and vertical directions. However, Lin \& Pierrehumbert (1987) showed that these three conditions can be satisfied contemporaneously only for the trivial case of a solid body rotation. Later, Fung (1992) considered the instability between two superimposed uniformly rotating vortex columns with different densities and angular velocities. He obtained a stability condition equivalent to that for two parallel streams of different velocity and densities.

However, the latter configuration is strongly idealized and it is unknown whether a shear instability can be triggered by the vertical shear present in a pancake vortex. In order to answer this question, we have investigated numerically the stability of a pancake vortex with a Gaussian vorticity profile in both radial and vertical directions which should be stable with respect to the radial shear instability. We shall show that a shear instability due to the vertical shear cannot occur because a gravitational instability appears much before when decreasing the aspect ratio.

The paper is organized as follows: in $\S 2$ we define the base state and describe the numerical method. The numerical results are presented in $\S 3$ and compared with theoretical and experimental results in $\S 4$. Section 5 generalizes the results to other velocity profiles while the stability criteria for the centrifugal instability will be discussed in $\S 6$. Section 7 includes concluding remarks. 

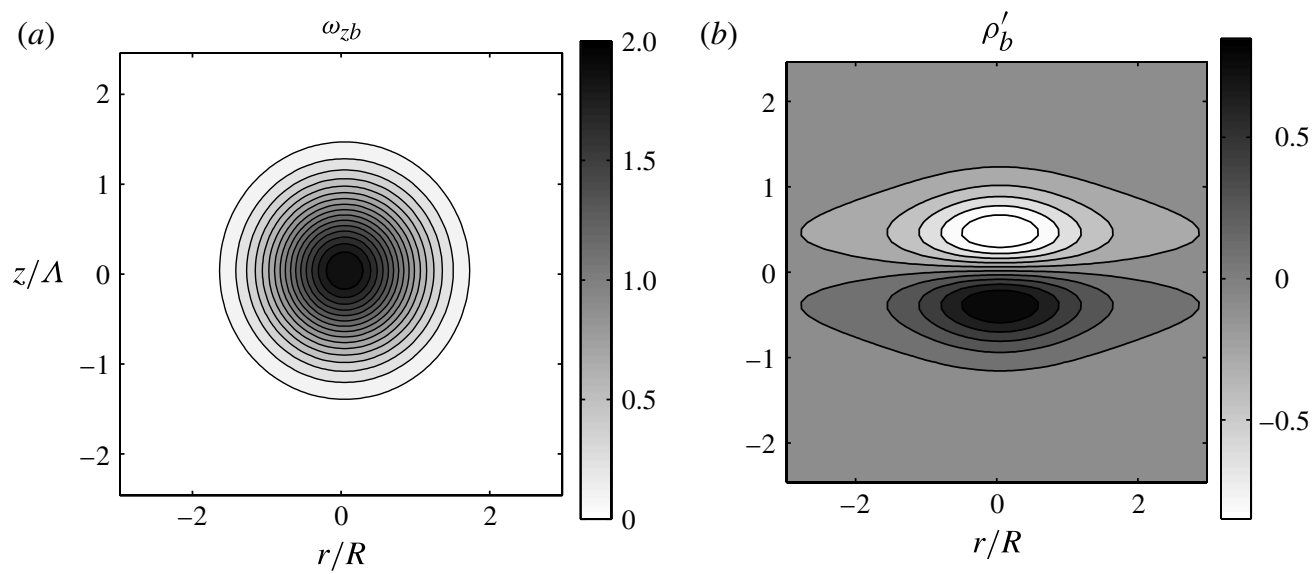

FIGURE 1. (a) Vertical vorticity $\omega_{z b}$ and $(b)$ perturbation density $\rho_{b}^{\prime}$ of the base state.

\section{Problem formulation}

\subsection{The base state}

We consider as basic flow a pancake vortex with velocity components $\left[0, u_{\theta b}(r, z), 0\right]$ in cylindrical coordinates $(r, \theta, z)$. The azimuthal velocity is chosen to have a Lamb-Oseen profile in the radial direction and a Gaussian profile in the vertical direction

$$
u_{\theta b}=\frac{\Gamma}{2 \pi}\left[\frac{1-\mathrm{e}^{-r^{2} / R^{2}}}{r}\right] \mathrm{e}^{-z^{2} / \Lambda^{2}}
$$

where $R$ is the characteristic radius, $\Lambda$ is the characteristic vertical size and $\Gamma$ is the circulation at the midplane $z=0$. The corresponding vertical vorticity $\omega_{z b}$ is Gaussian in both radial and vertical directions (see figure $1 a$ )

$$
\omega_{z b}=\frac{\Gamma}{\pi} \mathrm{e}^{-r^{2} / R^{2}-z^{2} / \Lambda^{2}} .
$$

Thus, the radial profile of vorticity is single-signed and the vortex should be stable with respect to a 'radial' two-dimensional shear instability according to the Rayleigh inflection point theorem. This differs from the pancake vortex with Gaussian angular velocity considered by Beckers et al. (2001) and Bonnier et al. (2000). Although a columnar Lamb-Oseen vortex is stable with respect to the centrifugal instability, the profile (2.1) is marginally centrifugally unstable as discussed in $\S 6$. The vortex (2.1) is a steady solution of the incompressible Euler equations with the Boussinesq approximation. The momentum equations in the radial and vertical directions read

$$
\begin{gathered}
\frac{u_{\theta b}^{2}}{r}-\frac{1}{\rho_{0}} \frac{\partial p_{b}}{\partial r}=0, \\
\frac{1}{\rho_{0}} \frac{\partial p_{b}}{\partial z}+\frac{g}{\rho_{0}} \rho_{b}^{\prime}=0,
\end{gathered}
$$

where $p_{b}$ is the pressure, $g$ the gravity and the total density $\rho_{b}$ has been decomposed as follows:

$$
\rho_{b}=\rho_{0}+\bar{\rho}(z)+\rho_{b}^{\prime}(\boldsymbol{x}),
$$


where $\rho_{0}$ is a reference density, $\bar{\rho}(z)$ the mean density profile and $\rho_{b}^{\prime}(\boldsymbol{x})$ a perturbation density due to the vortex. By combining (2.3), one obtains the so-called wind thermal relation

$$
\frac{\partial}{\partial z}\left(\frac{u_{\theta b}^{2}}{r}\right)=-\frac{g}{\rho_{0}} \frac{\partial \rho_{b}^{\prime}}{\partial r}
$$

which shows that the vertical gradient of azimuthal velocity implies a density perturbation $\rho_{b}^{\prime}$. By integrating (2.5), with the boundary condition $\rho_{b}^{\prime} \rightarrow 0$ as $r \rightarrow \infty$ one obtains

$$
\rho_{b}^{\prime}(r / R, z / \Lambda)=\frac{\rho_{0}}{g} \frac{\Gamma^{2}}{\pi^{2} \Lambda R^{2}} \frac{z}{\Lambda} \mathrm{e}^{-2 z^{2} / \Lambda^{2}}\left[\frac{-\left(\mathrm{e}^{-r^{2} / R^{2}}-1\right)^{2}}{2 r^{2} / R^{2}}+E_{1}\left(2 r^{2} / R^{2}\right)-E_{1}\left(r^{2} / R^{2}\right)\right]
$$

where $E_{1}$ is the exponential integral function defined as $E_{1}(\xi)=\int_{\xi}^{\infty} \mathrm{e}^{-\eta} / \eta \mathrm{d} \eta$ (Abramowitz \& Stegun 1970). As seen in figure 1(b), the density perturbation $\rho_{b}^{\prime}$ is negative above the midplane $z=0$ and positive below. This corresponds to a pinching of the isopycnal surfaces ( $\rho_{b}=$ const.) toward the minimum of pressure $p_{b}$ existing at the vortex centre.

\subsection{Numerical method of the three-dimensional stability analysis}

In order to study the stability of the base flow, the Navier-Stokes equations under the Boussinesq approximation are linearized

$$
\begin{gathered}
\frac{\partial \boldsymbol{u}}{\partial t}+\boldsymbol{\omega}_{b} \times \boldsymbol{u}+\boldsymbol{\omega} \times \boldsymbol{u}_{b}=-\nabla\left(\frac{p}{\rho_{0}}+\boldsymbol{u} \cdot \boldsymbol{u}_{b}\right)-\frac{g}{\rho_{0}} \rho \boldsymbol{e}_{z}+v \Delta \boldsymbol{u}, \\
\boldsymbol{\nabla} \cdot \boldsymbol{u}=0, \\
\frac{\partial \rho}{\partial t}+\boldsymbol{u} \cdot \nabla \rho_{b}+\boldsymbol{u}_{b} \cdot \nabla \rho=\kappa \Delta \boldsymbol{\rho}+N^{2} \frac{\rho_{0}}{g} u_{z},
\end{gathered}
$$

where $\boldsymbol{u}(\boldsymbol{x}, t), \boldsymbol{\omega}(\boldsymbol{x}, t), \rho(\boldsymbol{x}, t), p(\boldsymbol{x}, t)$ are infinitesimal perturbations of the velocity, vorticity, density and pressure, $N=\sqrt{-g / \rho_{0} \mathrm{~d} \bar{\rho} / \mathrm{d} z}$ is the Brunt-Väisälä frequency assumed constant, $v$ is the viscosity, $\kappa$ the diffusivity of the stratifying agent and $\boldsymbol{e}_{z}$ is the vertical unit vector pointing upward.

The Reynolds number, horizontal and vertical Froude numbers and Schmidt number are defined as

$$
R e=\frac{\Gamma}{2 \pi \nu}, \quad F_{h}=\frac{\Gamma}{2 \pi N R^{2}}, \quad F_{v}=\frac{F_{h}}{\alpha}, \quad S c=\frac{v}{\kappa}
$$

respectively, where $\alpha=\Lambda / R$ is the aspect ratio of the pancake vortex.

In (2.7), we have neglected the diffusion of the base state as classically done in stability analysis. This assumption is valid provided that the growth rate of the instabilities are large compared with the viscous damping of the base state.

The linearized equations (2.7) are integrated using a pseudospectral method in Cartesian coordinates $(x, y, z)$ with periodic boundary conditions (Delbende, Chomaz \& Huerre 1998; Deloncle et al. 2008). The velocity, vorticity, density and pressure perturbations are expressed in Fourier space after application of the three-dimensional Fourier transform

$$
[\hat{\boldsymbol{u}} ; \hat{\boldsymbol{\omega}} ; \hat{\rho} ; \hat{p}]\left(k_{x}, k_{y}, k_{z}, t\right)=\iiint[\boldsymbol{u} ; \boldsymbol{\omega} ; \rho ; p](x, y, z, t) \mathrm{e}^{-\mathrm{i} \boldsymbol{k} \cdot \boldsymbol{x}} \mathrm{d} x \mathrm{~d} y \mathrm{~d} z
$$


where $\mathrm{i}=\sqrt{-1}, k_{x}, k_{y}, k_{z}$ are the Cartesian components of the wavenumber $\boldsymbol{k}$ and the hat denotes the Fourier transform.

In spectral space, equations (2.7) become

$$
\begin{gathered}
\frac{\partial \hat{\boldsymbol{u}}}{\partial t}=\boldsymbol{P}(\boldsymbol{k})\left(\widehat{\boldsymbol{u} \times \boldsymbol{\omega}_{b}}+\widehat{\boldsymbol{\omega} \times \boldsymbol{u}_{b}}-\frac{g}{\rho_{0}} \hat{\rho} \boldsymbol{e}_{z}\right)-v \boldsymbol{k}^{2} \hat{\boldsymbol{u}} \\
\frac{\partial \hat{\rho}}{\partial t}=-\mathrm{i} \boldsymbol{k} \cdot\left(\widehat{\rho_{b} \boldsymbol{u}}+\widehat{\boldsymbol{u}_{b} \rho}\right)+N^{2} \frac{\rho_{0}}{g} \hat{\boldsymbol{u}}_{z}-\kappa \boldsymbol{k}^{2} \hat{\rho}
\end{gathered}
$$

The tensor $\boldsymbol{P}(\boldsymbol{k})$ with components $P_{i j}=\delta_{i j}-k_{i} k_{j} / \boldsymbol{k}^{2}$, is the projection on the space of divergence-free fields to enforce the continuity equation $\boldsymbol{k} \cdot \hat{\boldsymbol{u}}=0$. The time integration of the nonlinear terms of (2.10) is carried out with the fourth-order Runge-Kutta scheme while the viscous and diffusive terms are integrated exactly.

The domain size $L_{x} \times L_{y} \times L_{z}$ is chosen as $6 R \times 6 R \times 4 R$ with $N_{x} \times N_{y} \times N_{z}=$ $128 \times 128 \times 64$ collocation points. The time step is set to $\delta t=0.005 \cdot\left(2 \pi R^{2} / \Gamma\right)$. The perturbations are initialized by divergence-free white noise. The most unstable mode emerges after a sufficient integration time

$$
\lim _{t \rightarrow \infty}[\boldsymbol{u}(\boldsymbol{x}, t), \rho(\boldsymbol{x}, t)]=[\tilde{\boldsymbol{u}}(\boldsymbol{x}), \tilde{\rho}(\boldsymbol{x})] \mathrm{e}^{\sigma t}+\text { c.c. },
$$

where $\sigma$ is the eigenvalue of the overall dominant eigenmode and c.c. denotes the complex conjugate. However, since the basic flow is axisymmetric, each azimuthal mode evolves independently of the other and it is possible to retrieve the dominant eigenmode for each azimuthal wavenumber $m$ by applying a modal decomposition (Delbende et al. 1998). First, the velocity in Cartesian coordinates $\boldsymbol{u}(\boldsymbol{x}, t)$ is interpolated on a grid of cylindrical coordinates $(r, \theta, z)$ via a 'slow' reverse Fourier transform

$$
\boldsymbol{u}(r, \theta, z, t)=\frac{1}{(2 \pi)^{3}} \iiint \hat{\boldsymbol{u}}\left(k_{x}, k_{y}, k_{z}, t\right) \mathrm{e}^{\mathrm{i}\left(k_{x} r \cos \theta+k_{y} r \sin \theta+k_{z} z\right)} \mathrm{d} k_{x} \mathrm{~d} k_{y} \mathrm{~d} k_{z}
$$

with $N_{r} \times N_{\theta}=64 \times 32$ points in the radial and azimuthal directions. The velocity in cylindrical coordinates is then given by $\boldsymbol{v}=\left(v_{r}, v_{\theta}, v_{z}\right)=\left(u_{x} \cos \theta+u_{y} \sin \theta,-u_{x} \sin \theta+\right.$ $\left.u_{y} \cos \theta, u_{z}\right)$. Each azimuthal component $m$ is then isolated by means of the azimuthal Fourier transform

$$
\boldsymbol{v}_{m}(r, z, t)=\int_{0}^{2 \pi} \boldsymbol{v}(r, \theta, z, t) \mathrm{e}^{-\mathrm{i} m \theta} \mathrm{d} \theta .
$$

Owing to the symmetry of the base state with respect to the horizontal midplane $z=0$, the normal modes separate further into two independent classes, symmetric and antisymmetric, defined as

$$
\begin{aligned}
& {\left[v_{r m}, v_{\theta m}, v_{z m}, \rho_{m}\right](r, z, t)=\left[v_{r m}, v_{\theta m},-v_{z m},-\rho_{m}\right](r,-z, t),} \\
& {\left[v_{r m}, v_{\theta m}, v_{z m}, \rho_{m}\right](r, z, t)=\left[-v_{r m},-v_{\theta m}, v_{z m}, \rho_{m}\right](r,-z, t),}
\end{aligned}
$$

respectively. The dominant eigenmode and eigenvalue are then retrieved for each azimuthal wavenumber $m$ and each symmetry as in (2.11), i.e.

$$
\lim _{t \rightarrow \infty}\left[\boldsymbol{v}_{m}(r, z, t), \rho_{m}(r, z, t)\right]=\left[\tilde{\boldsymbol{v}}_{m}(r, z), \tilde{\rho}_{m}(r, z)\right] \mathrm{e}^{\sigma t} .
$$

Without loss of generality, the azimuthal wavenumber will be assumed to be positive because of the symmetry $\sigma(m)=\sigma^{*}(-m)$. The accuracy and the convergence of the results have been checked by varying the size of the computational box and the 


\begin{tabular}{lclll}
$L_{x} \times L_{y} \times L_{z}$ & $N_{x} \times N_{y} \times N_{z}$ & $\delta t$ & \multicolumn{2}{c}{$\sigma_{r}(m=1)$} \\
& & & Symmetric & Antisymmetric \\
$4 \times 4 \times 4$ & $64 \times 64 \times 64$ & 0.01 & 0.301033 & 0.301034 \\
$4 \times 4 \times 8$ & $128 \times 128 \times 256$ & 0.005 & 0.301037 & 0.30104 \\
$8 \times 8 \times 4$ & $256 \times 256 \times 128$ & 0.005 & 0.301039 & 0.301041 \\
$6 \times 6 \times 4$ & $128 \times 128 \times 64$ & 0.005 & 0.301029 & 0.301042
\end{tabular}

TABLE 1. The different growth rates $\sigma_{r}$ relative to $m=1$ obtained using different resolutions $N_{x} \times N_{y} \times N_{z}$ and box sizes $L_{x} \times L_{y} \times L_{z}$ for both symmetric and antisymmetric modes for $\alpha=0.85, R e=500, F_{h}=1$ and $S c=1$.
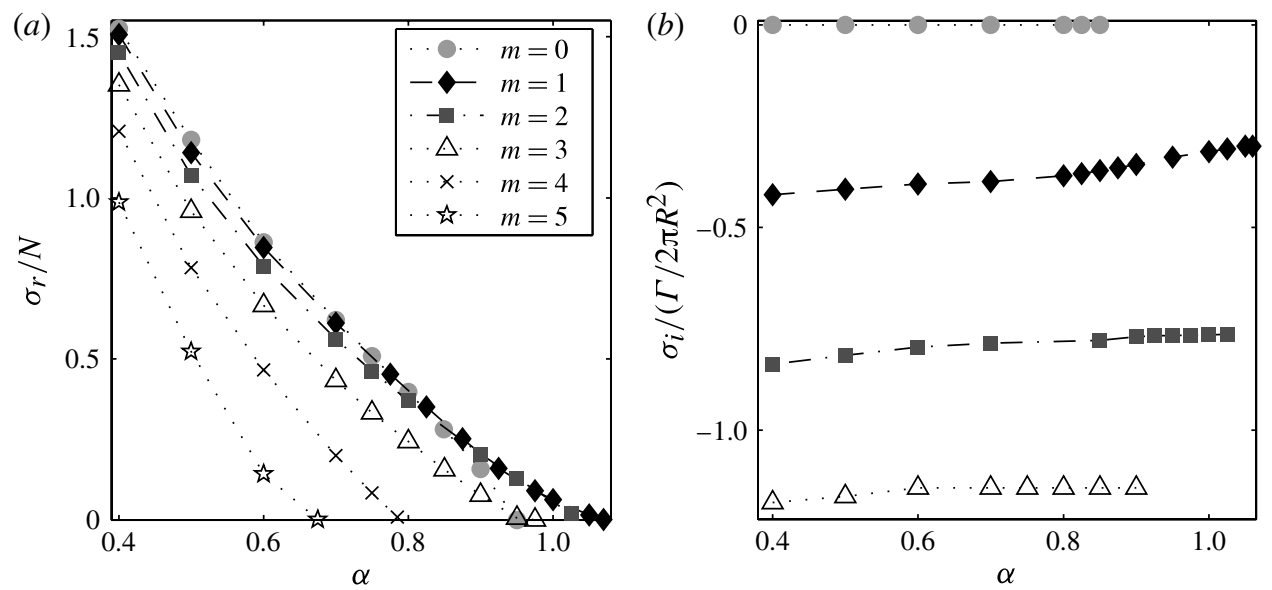

FIGURE 2. Stability characteristics of the pancake vortex for $F_{h}=1, R e=500$ and $S c=1$. (a) Non-dimensional growth rate $\sigma_{r} / N$ and $(b)$ frequency $\sigma_{i} / \Gamma / 2 \pi R^{2}$ as a function of the aspect ratio $\alpha$ for different azimuthal wavenumbers $m$.

resolution. As seen in table 1, the growth rate varies by less than $0.1 \%$ in the cases tested.

\section{Numerical results}

\subsection{Effect of the aspect ratio}

Typical results are displayed in figure 2 for $F_{h}=1, R e=500$ and $S c=1$. The growth rates $\sigma_{r}$ (figure $2 a$ ) are plotted versus the aspect ratio $\alpha$ for the first six azimuthal wavenumbers $m=0$ to $m=5$ of the symmetric class. The results relative to the antisymmetric modes are not presented because they have very close growth rates (see table 1 for $\alpha=0.85$ ). As seen in figure 2(a), the pancake vortex becomes more and more unstable when the aspect ratio decreases from $\alpha \sim 1.07$ with an increasing number of unstable azimuthal wavenumbers. The corresponding frequency (figure $2 b$ ) is almost independent of $\alpha$ and is proportional to $m$ demonstrating that each azimuthal wavenumber rotates at the same phase velocity $\sigma_{i} / m$.

The growth rates of the azimuthal modes $m=0,1,2$ are close so that the most unstable azimuthal mode is either $m=0,1,2$ depending on the aspect ratio $\alpha$. When $\alpha \leqslant 0.75$, the growth rate monotonically decreases with $m$. The axisymmetric mode is 

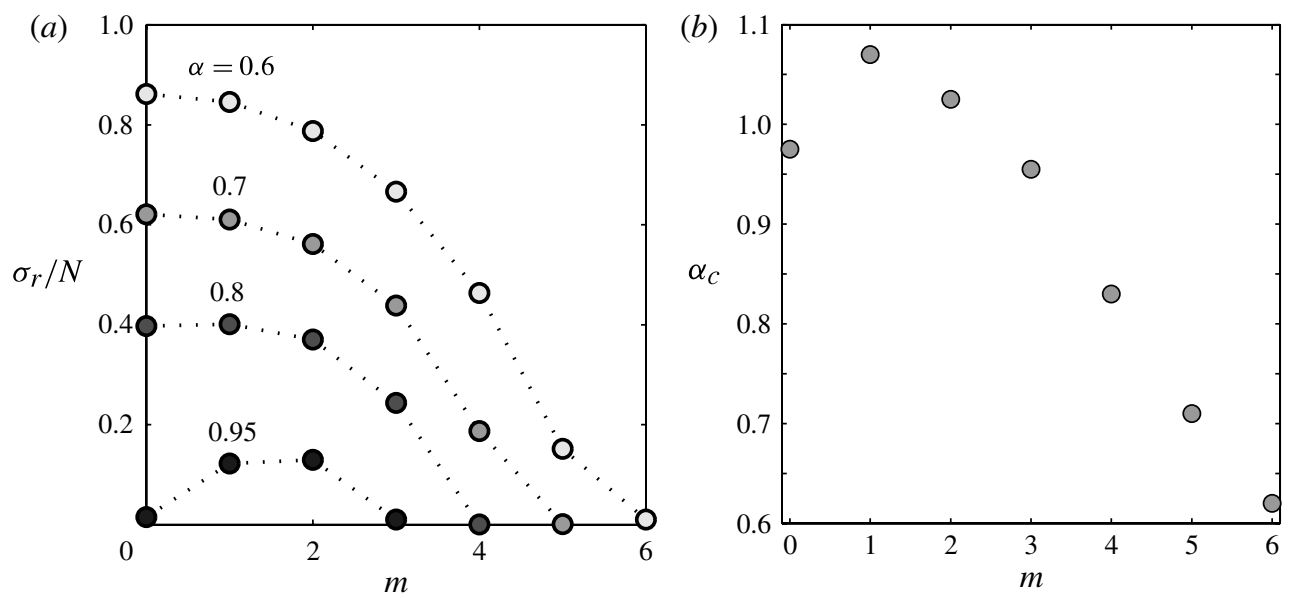

FIGURE 3. Non-dimensional growth rate $\sigma_{r} / N$ for different aspect ratios $\alpha(a)$ and critical aspect ratio $\alpha_{c}(b)$ as a function of the azimuthal wavenumber $m\left(F_{h}=1, \operatorname{Re}=500, S c=1\right)$.

therefore dominant (figure $2 a$ ) but only marginally: the growth rate for $m=1$ is very close to that for $m=0$.

When $\alpha>0.75$, the two modes $m=1,2$ become more dangerous than $m=0$ and have almost the same growth rate. Although they are undistinguishable in figure 2, the mode $m=1$ is in fact slightly more unstable than $m=2$ for $0.75<\alpha<0.85$ (see figure $3 a$ ) and near the threshold $\alpha \sim 1.07$. In contrast, higher azimuthal modes $m \geqslant 3$ are always less unstable with a growth rate decreasing with $m$. The critical aspect ratio is plotted in figure $3(b)$ as a function of $m$. The modes $m=0,1,2$ become stable for $\alpha_{c}=0.95,1.07,1.03$, respectively. The critical aspect ratio then decreases monotonically for larger $m$. The perturbation vertical vorticity $\omega_{z m}(r, z)$ and vertical velocity $v_{z m}(r, z)$ for the modes $m=0,1,2$ for $\alpha=0.85$ are displayed in figures 4 and 5 , respectively. We see that the perturbation vertical vorticity is localized in the upper and lower midplanes and is maximum near $z / \Lambda \approx \pm 0.66$ for each $m$. This is close to the location at which the vertical gradient of the base state velocity is maximal, namely at $z / \Lambda= \pm 1 / \sqrt{2}= \pm 0.707$. In contrast, the radial size of the perturbations increases with $m$ : the radius of the perturbation vertical vorticity maxima (real part) $r_{m}$ increases with $m: r_{0} \approx 0, r_{1} \approx 0.2 R, r_{2} \approx 0.33 R, r_{3} \approx 0.36 R$ (the latter case is not shown). As seen in figure 5 , the perturbation vertical velocity reaches its maximum around $z / \Lambda \approx 0.9$ close to the location $z / \Lambda \sim \sqrt{3 / 4}=0.866$ where the vertical gradient of the base state density $\partial \rho_{b} / \partial z$ is maximum.

In order to have a clearer view of the perturbation structure, the vertical vorticity isosurfaces of the perturbation for $m=1$ and $\alpha=1$ are displayed in three dimensions in figure 6. The modes rotate steadily at frequency $\sigma_{i}$ without change of shape, i.e. it is given at any time $t$ by $\omega_{z m}(r, \theta, z, t)=\pi\left[\operatorname{Re}\left(\omega_{z m}(r, z)\right) \cos \left(m \theta+\sigma_{i} t+\varphi\right)-\right.$ $\left.\operatorname{Im}\left(\omega_{z m}(r, z)\right) \sin \left(m \theta+\sigma_{i} t+\varphi\right)\right]$, where $\varphi$ is a constant phase.

Since the perturbation is located in two distinct regions in the upper and lower parts of the base flow, the perturbation in each of these two regions behave almost independently. This explains why the growth rate of symmetric and antisymmetric modes are very close as mentioned previously. 

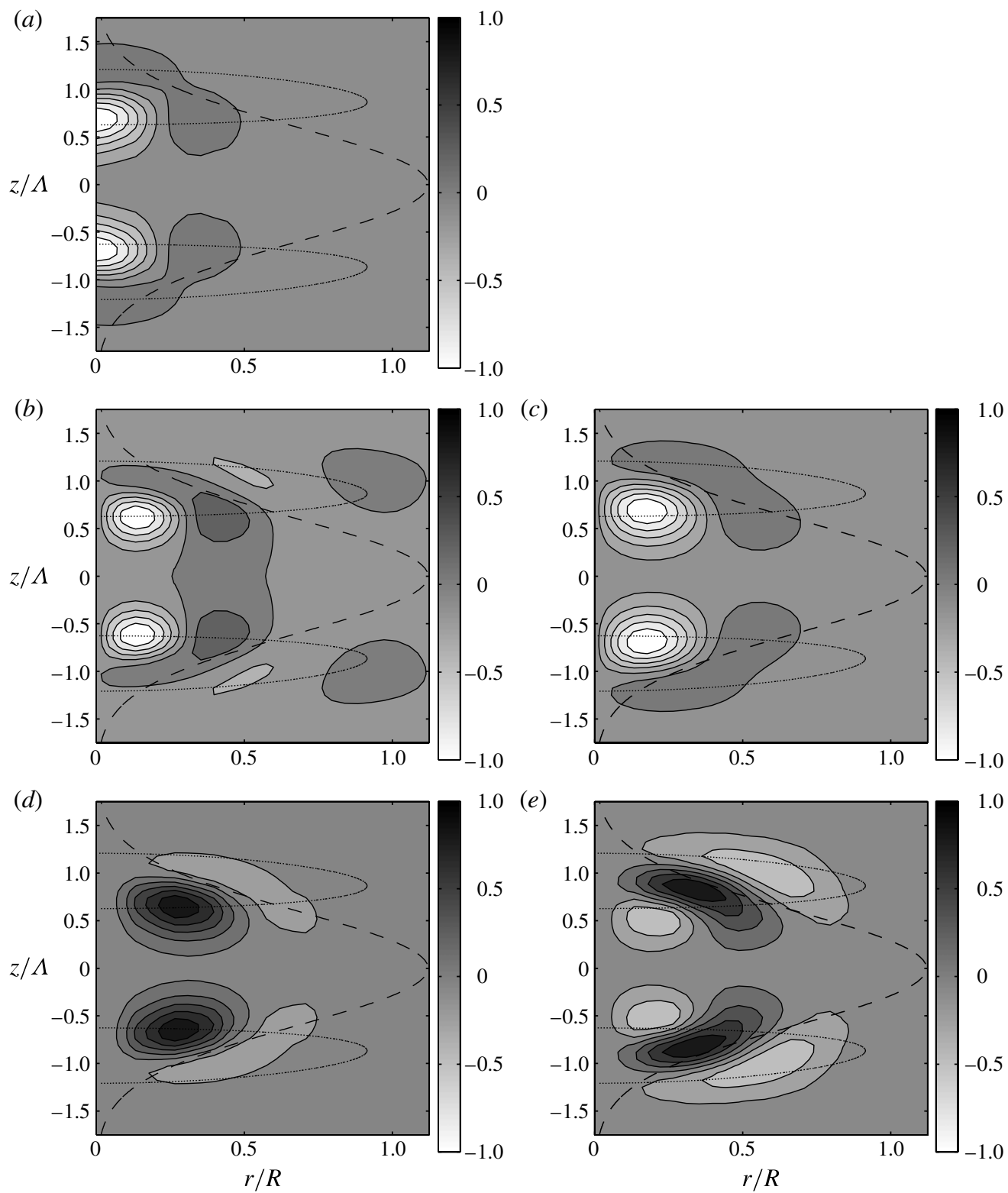

FIGURE 4. Real $(a, b, d)$ and imaginary $(c, e)$ part of the vertical vorticity perturbation $\omega_{z m}(r, z) /\left|\omega_{z m}\right|_{\max }$ for $m=0(a), m=1(b, c)$ and $m=2(d, e)\left(\alpha=0.85, R e=500, F_{h}=1\right.$, $S c=1)$. The dashed lines correspond to the location of maximal azimuthal velocity given by $r / R=1.1209 \mathrm{e}^{-z^{2} / \Lambda^{2}}$. The dotted lines delimit the regions where the total vertical density gradient of the base state is positive.

\subsection{Effect of the horizontal Froude number}

The effect of the horizontal Froude number is now investigated. In figure 7, the growth rate $\sigma_{r}(a)$ and the frequency $\sigma_{i}(b)$ of the azimuthal wavenumber $m=1$ are plotted versus $\alpha / F_{h}$, i.e. the inverse of the vertical Froude number $1 / F_{v}$, for different 

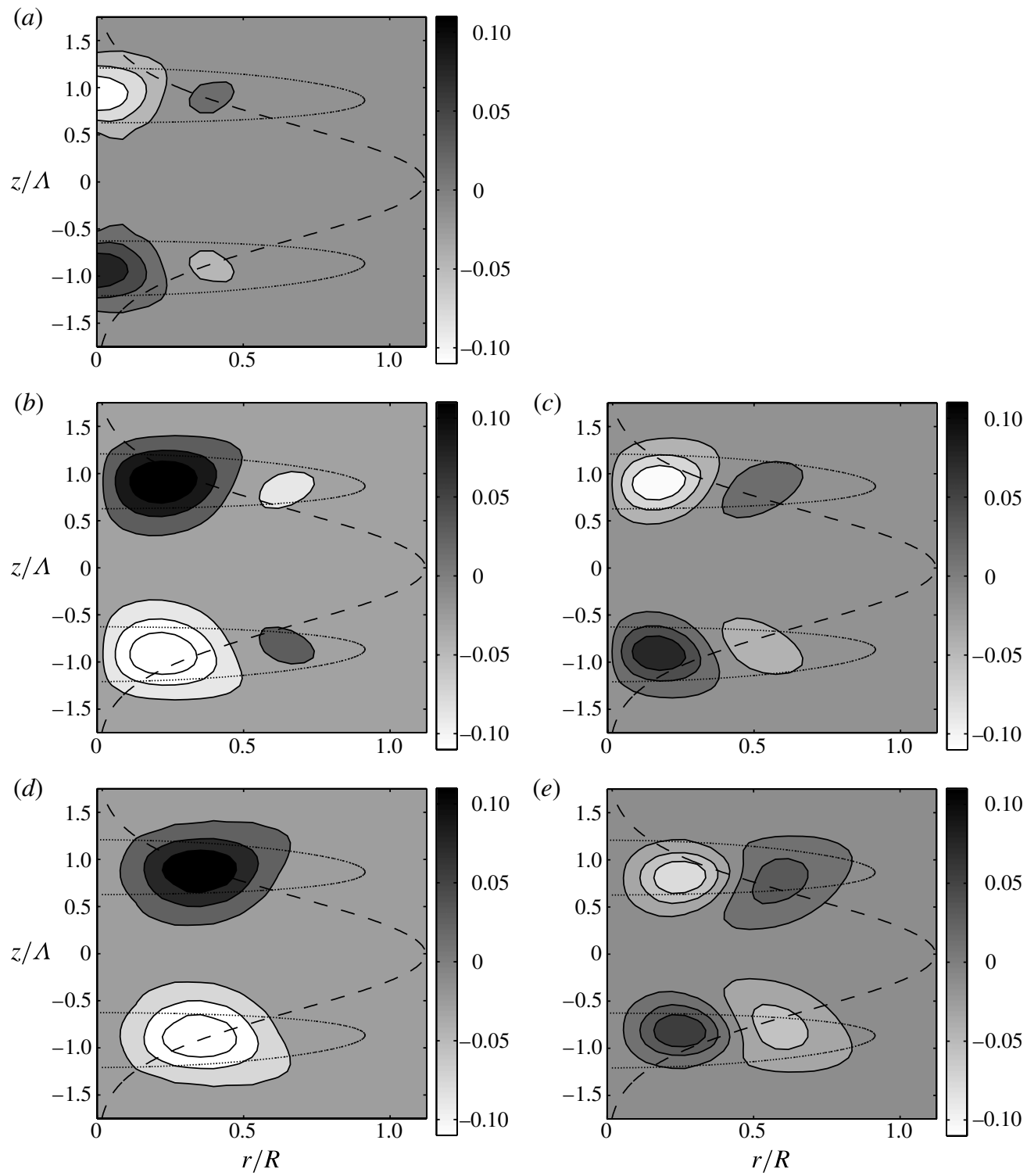

FIGURE 5. Real $(a, b, d)$ and imaginary $(c, e)$ part of the vertical velocity perturbation $v_{z m}(r, z) /\left|\omega_{z m}\right|_{\max }$ for $m=0(a), m=1(b, c)$ and $m=2(d, e)\left(\alpha=0.85, R e=500, F_{h}=1\right.$, $S c=1)$. The dashed line corresponds to the location of maximal azimuthal velocity given by $r / R=1.1209 \mathrm{e}^{-z^{2} / \Lambda^{2}}$. The dotted lines delimit the regions where the total vertical density gradient of the base state is positive.

values of the horizontal Froude number. Like for $F_{h}=1, R e=500$ (figure 2), the growth rates of the azimuthal modes $m=0,2$ are very close regardless of the Froude number $F_{h}$ and are therefore not shown. We can see that the growth rate $\sigma_{r}$ scaled by the Brunt-Väisälä frequency $N$ is almost superposed for $F_{h}=1.5$ and $F_{h}=1$ for $R e=500$. In contrast, the growth rate curves for $F_{h}=0.5$ and $F_{h}=0.25$ for $R e=500$ are slightly lower and decrease continuously with the Froude number for a 


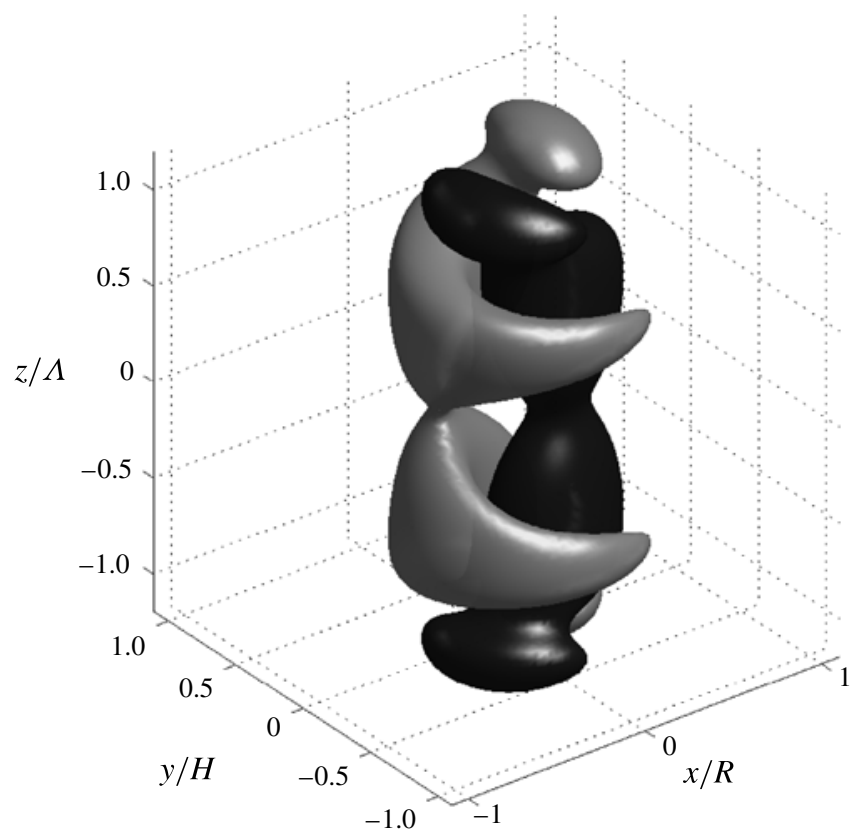

FIGURE 6. Three-dimensional view of the vertical vorticity perturbation for $m=1, \alpha=1.0$, $F_{h}=1, R e=500$ and $S c=1$. The isosurfaces correspond to the values $\pm 45 \%$ of the maximum vorticity.
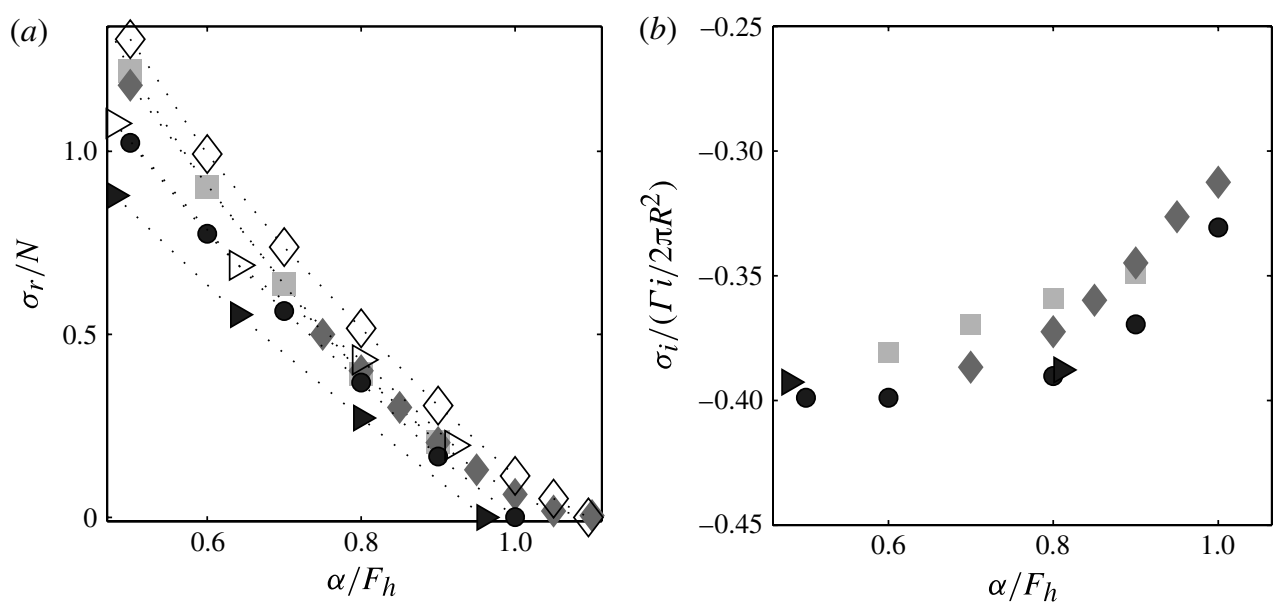

FIGURE 7. Non-dimensional growth rate $\sigma_{r} / N(a)$ and frequency $\sigma_{i} / \Gamma / 2 \pi R^{2}(b)$ of the mode $m=1$ as a function of $\alpha / F_{h}$ for different values of the Froude number and the Reynolds number for $S c=1: \backsim F_{h}=1.5 ; \diamond F_{h}=1 ; \bullet F_{h}=0.5 ; \triangleright F_{h}=0.25$ for $R e=500 ; \triangleright F_{h}=0.25$; $\diamond F_{h}=1.0$ for $\operatorname{Re}=1000$.

given value of $\alpha / F_{h}$. However, if the Reynolds number is increased to $\operatorname{Re}=1000$ for $F_{h}=0.25$, we see that the scaled growth rate is again close to those for $0.5 \leqslant F_{h} \leqslant 1.5$, $R e=500$. Similarly, the results for $F_{h}=1$ and $R e=1000$ are slightly higher than 


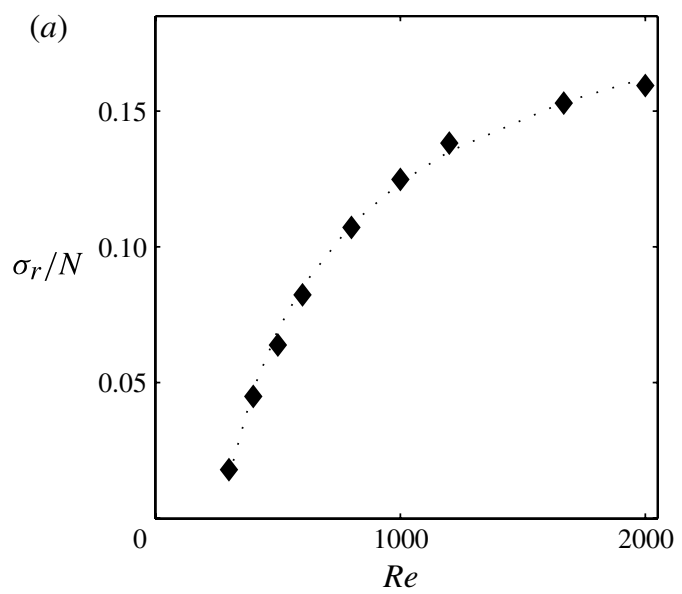

(b)

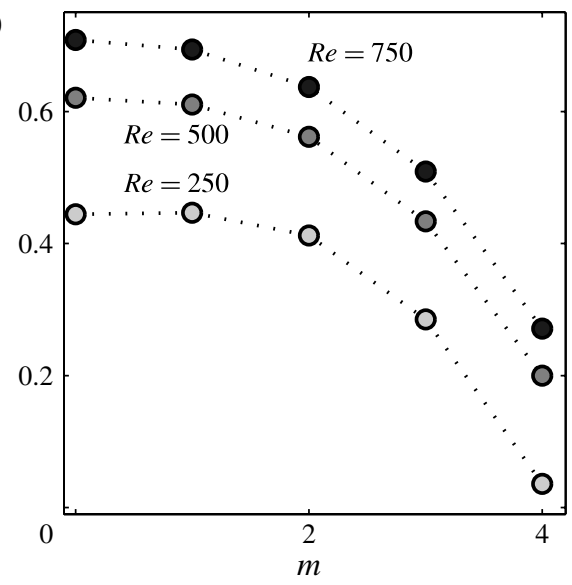

FIGURE 8. Effects of the Reynolds number. Non-dimensional growth rate $\sigma_{r} / N$ as a function of (a) the Reynolds number $\operatorname{Re}\left(m=1, \alpha=1.0, F_{h}=1, S c=1\right)$ and of $(b)$ the azimuthal wavenumber $m$ for $\alpha=0.7, F_{h}=1, S c=1$ and three Reynolds numbers $R e=250,500$ and 750. The dotted line in $(a)$ shows the relation $\sigma_{r} / N=\sigma_{i n v} / N-C / \sqrt{R e}$ with $\sigma_{i n v} \approx 0.255$ and $C \approx 4.15$.

those for $F_{h}=1, R e=500$ but the critical aspect ratio is still the same $\alpha_{c} \sim 1.1$. These results suggest that the scaled growth rate depends only on $F_{h} / \alpha$ provided that diffusive effects are sufficiently small. Viscous effects will be investigated further in the next section. Similarly, the frequency $\sigma_{i}$ scaled by the turnover frequency $\Gamma / 2 \pi R^{2}$ depends almost only on $\alpha / F_{h}$ (figure $7 b$ ).

\subsection{Effect of the Reynolds number}

Figure $8(a)$ shows the dependence of the growth rate of the azimuthal wavenumber $m=1$ on the Reynolds number for a fixed aspect ratio and Froude number. The growth rate increases with $R e$ and follows approximately the relation $\sigma_{r} \approx$ $\sigma_{i n v}-C N / \sqrt{R e}$, with $\sigma_{i n v} \approx 0.255$ and $C \approx 4.15$, as highlighted by the dotted line. This confirms that the growth rate tends to a constant value $\sigma_{i n v}$ in the inviscid limit. The particular dependence with $R e$ will be explained in $\$ 4.2$. The effect of the Reynolds number on the growth rate of each azimuthal wavenumber is displayed in figure $8(b)$. An increase of the Reynolds number increases the growth rate of each azimuthal mode and thus widens the band of unstable azimuthal wavenumber $m$.

\subsection{Effect of the Schmidt number}

Finally, we have investigated another value of the Schmidt number: $S c=7$. This value which is representative of temperature stratified water remains accessible numerically with the same numerical resolution as for $S c=1$. As seen in figure 9, the scaled growth rate $\sigma_{r} / N$ of the most amplified azimuthal wavenumbers $m=0,1,2\left(F_{h}=1\right.$, $R e=500$ ) are slightly larger than for $S c=1$. The value $S c=700$ corresponding to salt stratified water has not been investigated numerically but it will be discussed on the basis of theoretical predictions in $\S 4.2$. 


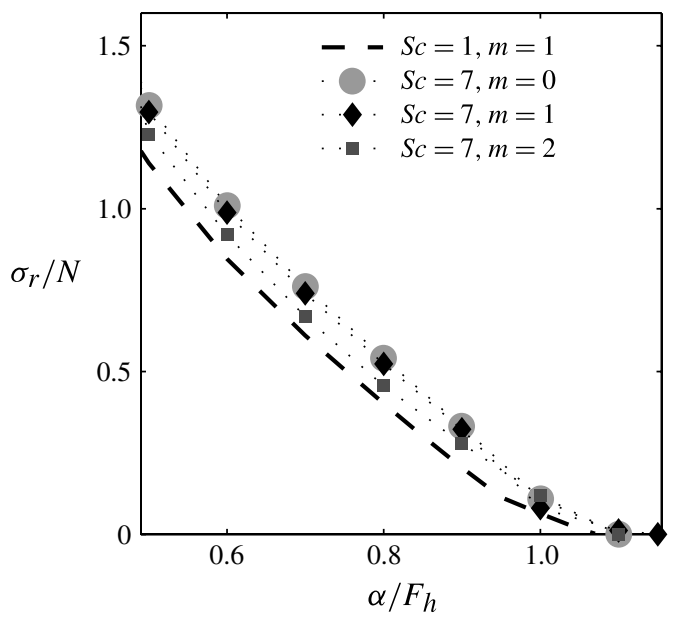

FIGURE 9. Non-dimensional growth rate $\sigma_{r} / N$ as a function of $\alpha / F_{h}$ for a Schmidt number $S c=7$ and for the azimuthal wavenumbers $m=0,1,2\left(F_{h}=1, \operatorname{Re}=500\right)$. The dashed line represents the numerical results for $S c=1$ and $m=1$ for reference.

\section{Interpretation and comparison with theory and experiments}

\subsection{Instability conditions}

In order to determine the nature of the instability, it is interesting to look at the conditions for the gravitational and shear instabilities in the inviscid limit. The gravitational instability is expected to occur when the vertical gradient of the total density is positive somewhere, i.e.

$$
\frac{\partial \rho_{b}}{\partial z}=\frac{\partial \rho_{b}^{\prime}}{\partial z}-N^{2} \frac{\rho_{0}}{g}>0 .
$$

Since the vertical gradient of the base state density (2.6), is maximum at $r=0$ and $z / \Lambda= \pm \sqrt{3 / 4}$, the instability condition becomes

$$
\mathscr{K}_{2} \frac{F_{h}^{2}}{\alpha^{2}}-1>0, \quad \text { where } \mathscr{K}_{2}=8 \ln (2) \mathrm{e}^{-3 / 2} .
$$

Thus, gravitationally unstable regions appear when $\alpha<\alpha_{c} \equiv 1.11 F_{h}$. This is illustrated in figure $10(a)$, where the vertical profile of density for $F_{h}=1$ and $\alpha=0.85$ (dashed line) and $\alpha=1.2$ (solid line) are plotted along the vortex axis. It can be seen that the total density increases in the regions around $z / \Lambda= \pm \sqrt{3 / 4} \sim 0.87$ for $\alpha=0.85$ in contrast to $\alpha=1.2$. The gravitationally unstable regions are also indicated by dotted lines in figures 4 and 5. They correspond well to the locations of the perturbations.

The well-known necessary condition for the shear instability of a parallel inviscid shear flow is $0<\min (R i)<1 / 4$ where $R i$ is the Richardson number. Even if the basic flow is here not parallel, we have looked at this condition by computing the local Richardson number based on the vertical shear

$$
R i=\frac{-\frac{g}{\rho_{0}} \frac{\partial \rho_{b}}{\partial z}}{\left[\frac{\partial u_{\theta b}}{\partial z}\right]^{2}} .
$$



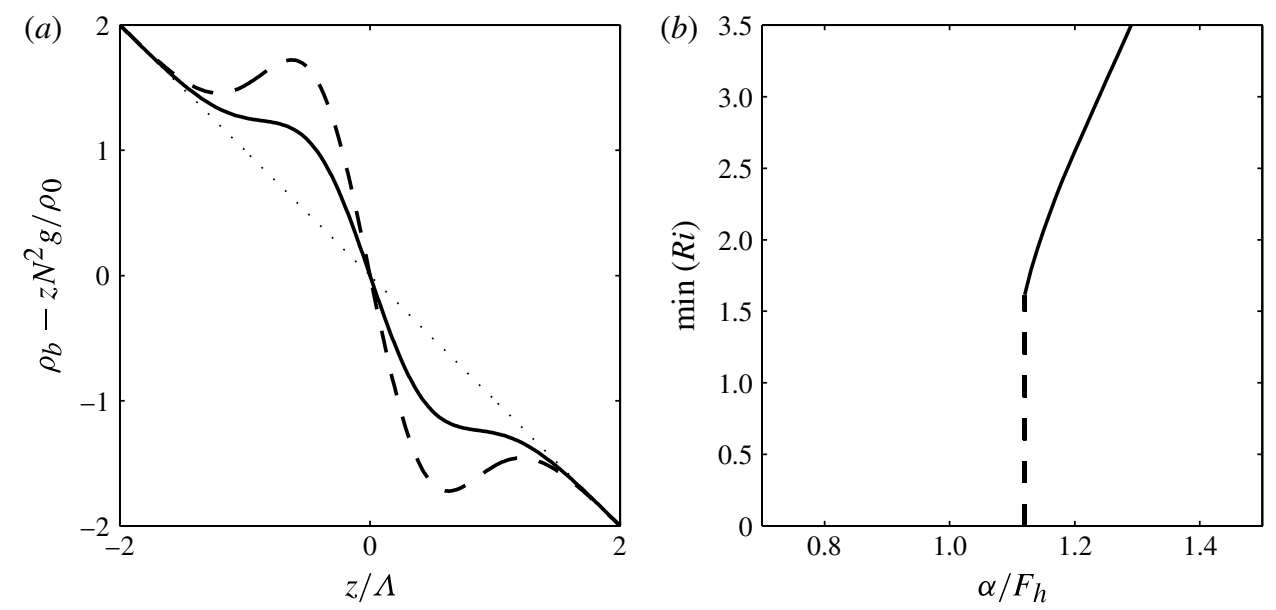

FIGURE 10. (a) Vertical profiles of the density of the base state for $F_{h}=1$ and for $\alpha=0.85$ (dashed line) and $\alpha=1.2$ (solid line). (b) Minimum of the local Richardson number as a function of $\alpha / F_{h}$.

Using (2.1) and (2.6), it can be written in the convenient form

$$
R i=\frac{\frac{\alpha^{2}}{F_{h}^{2}}+2\left(f f^{\prime}\right)^{\prime} \int_{\tilde{r}}^{\infty} \frac{h^{2}(\mathscr{R})}{\mathscr{R}} \mathrm{d} \mathscr{R}}{h^{2} f^{\prime 2}} .
$$

where $h(\tilde{r})=1 / \tilde{r}\left(1-\mathrm{e}^{-\tilde{r}^{2}}\right)$ and $f(\tilde{z})=\mathrm{e}^{-\tilde{z}^{2}}$, with $\tilde{r}=r / R$ and $\tilde{z}=z / \Lambda$. The prime denotes derivative with respect to $\tilde{z}$. This shows that the Richardson number depends only on the vertical Froude number $F_{v}=F_{h} / \alpha$ for a given shape of the velocity profile.

Figure 10(b) shows the minimum value of $R i$ as a function of $\alpha / F_{h}$. We can see that $\min (R i)$ is larger than 1.6 for $\alpha>1.11 F_{h}$ and then becomes abruptly negative for $\alpha<1.11 F_{h}$ since the maximum of the total density vertical gradient is then positive. Therefore, the instability condition for a vertical shear instability $0<\min (R i)<1 / 4$ is never satisfied.

Since the threshold for the gravitational instability $\alpha / F_{h} \sim 1.11$ is in very good agreement with the value found in the numerical stability analysis when viscous effects are small: $\alpha / F_{h} \sim 1.1$ (cf. figure 2), it is very likely that the dominant instability of the pancake vortex is a gravitational instability. In order to explain the properties of the instability, especially the azimuthal wavenumber selection, we will consider in the next section a uniformly rotating and unstably stratified layer with a constant or varying density gradient along the vertical.

\subsection{Linear stability analysis of an unstably stratified rotating layer}

We first consider an unstably stratified viscous fluid, with constant Brunt-Väisälä frequency $N_{u}$ (with $N_{u}^{2}=-g / \rho_{0} \partial \rho_{b}(z) / \partial z<0$ ) and in solid body rotation with a constant angular velocity $\Omega$. Such basic flow is identical to a fluid layer heated from below and rotating at a constant rate considered in many studies describing the effect of rotation on Rayleigh-Bénard convection (Chandrasekhar 1961). 
We rewrite in cylindrical coordinates $(r, \theta, z)$ the Boussinesq equation (2.7) linearized around such base flow and assume normal modes solutions of the form $\left[u_{r}, u_{\theta}, u_{z}, \rho, p\right]=\left[\hat{u}_{r}, \hat{u}_{\theta}, \hat{u}_{z}, \hat{\rho}, \hat{p}\right](r) \exp (\sigma t+\mathrm{i} m \theta+\mathrm{i} k z)+c c$, where $k$ is the vertical wavenumber. The disturbance equations then read

$$
\begin{aligned}
s \hat{u}_{r}-2 \Omega \hat{u}_{\theta} & =-\frac{1}{\rho_{0}} \frac{\mathrm{d} \hat{p}}{\mathrm{~d} r}+v\left[\Delta \hat{u}_{r}-\frac{\hat{u}_{r}}{r^{2}}-\frac{2 \mathrm{i} m}{r^{2}} \hat{u}_{\theta}\right] \\
s \hat{u}_{\theta}+2 \Omega \hat{u}_{r} & =-\frac{\mathrm{i} m}{\rho_{0}} \frac{\hat{p}}{r}+v\left[\Delta \hat{u}_{\theta}-\frac{\hat{u}_{\theta}}{r^{2}}+\frac{2 \mathrm{i} m}{r^{2}} \hat{u}_{r}\right] \\
s \hat{u}_{z}+g \frac{\hat{\rho}}{\rho_{0}} & =-\frac{\mathrm{i} k}{\rho_{0}} \hat{p}+v \Delta \hat{u}_{z} \\
s \hat{\rho}-\frac{\rho_{0}}{g} N_{u}^{2} \hat{u}_{z} & =\kappa \Delta \hat{\rho} \\
\frac{\mathrm{d} \hat{u}_{r}}{\mathrm{~d} r}+\frac{\hat{u}_{r}}{r}+\frac{\mathrm{i} m \hat{u}_{\theta}}{r}+\mathrm{i} k \hat{u}_{z} & =0
\end{aligned}
$$

where $s=\mathrm{i} m \Omega+\sigma$ and $\Delta=(1 / r) \mathrm{d} / \mathrm{d} r(r \mathrm{~d} / \mathrm{d} r)-m^{2} / r^{2}-k^{2}$. As shown in Chandrasekhar (1961), these equations can be rewritten in terms of $\hat{u}_{z}$ only

$$
(s-v \Delta)^{2}(s-\kappa \Delta) \Delta \hat{u}_{z}=4 \Omega^{2} k^{2}(s-\kappa \Delta) \hat{u}_{z}-N_{u}^{2}(s-v \Delta) \Delta_{h} \hat{u}_{z}
$$

where $\Delta_{h}=(1 / r) \mathrm{d} / \mathrm{d} r(r \mathrm{~d} / \mathrm{d} r)-m^{2} / r^{2}$. Therefore, solutions of the form $\hat{u}_{z} \propto \mathrm{J}_{m}(q r)$, where $\mathbf{J}_{m}$ is the Bessel function of the first kind of order $m$ and $q$ a constant, gives the dispersion relation

$$
\begin{aligned}
& {\left[s+v\left(q^{2}+k^{2}\right)\right]^{2}\left[s+\kappa\left(q^{2}+k^{2}\right)\right]\left(q^{2}+k^{2}\right)} \\
& \quad+4 \Omega^{2} k^{2}\left[s+\kappa\left(q^{2}+k^{2}\right)\right]+N_{u}^{2}\left[s+v\left(q^{2}+k^{2}\right)\right] q^{2}=0 .
\end{aligned}
$$

In the particular case $v=\kappa$, this dispersion relation reduces to a second-order polynomial which gives directly

$$
s=-v\left(q^{2}+k^{2}\right) \pm \mathrm{i} \sqrt{\frac{4 \Omega^{2} k^{2}+N_{u}^{2} q^{2}}{q^{2}+k^{2}}} .
$$

In the inviscid and diffusionless limit $v=0$ and for a stably stratified fluid $N_{u}^{2}>0$, equation (4.8) is the classical dispersion relation of inertia-gravity waves. For a given value of $q$, the frequency varies from $\sigma_{i}=-m \Omega \pm 2 \Omega$ for large vertical wavenumbers $k$ to $\sigma_{i}=-m \Omega \pm N_{u}$ for $k=0$.

However, when the fluid is unstably stratified $N_{u}^{2}<0$, we see that instability will occur in the range of vertical wavenumber

$$
k^{2}<\frac{-N_{u}^{2} q^{2}}{4 \Omega^{2}} .
$$

The maximum growth rate $\sigma_{r, \max }=\left|N_{u}\right|$ is obtained in the two-dimensional limit $k=0$ for any finite value of $q$. In the case of a viscous fluid within a bounded domain, the boundary conditions will impose lower bounds on the vertical and radial wavenumbers $k$ and $q$ whereas viscous and diffusive effects stabilize large wavenumbers. The selected wavenumbers will depend therefore on the size of the gravitationally unstable region of the pancake vortex and the Reynolds number. 
As shown in figure 5 for $\alpha=0.85 F_{h}$, the vertical velocity of the mode is confined in the vertical direction, i.e. its vertical size is approximately equal to the height $\delta_{z}$ of the gravitationally unstable region which is delimited by dotted lines. Therefore, the corresponding vertical wavenumber can be estimated to $k \approx \pi / \delta_{z}$. In contrast, the mode is concentrated radially and is smaller than the mean radius $\delta_{r}$ of the gravitationally unstable region. This feature is observed when $\alpha$ is sufficiently small, typically $\alpha \leqslant 0.9 F_{h}$. For larger aspect ratios, the modes tend to be confined both vertically and radially and even to overshoot in stable regions.

In order to compare the predictions of (4.8) to the numerical observations, we shall therefore assume that the vertical wavenumber is set to $k=\pi / \delta_{z}$ (with free boundary conditions) but the domain is radially unbounded so that the radial wavenumber $q$ is free and sets to the most amplified value $q_{\max }$. Since the total density gradient is not uniform in the pancake vortex, we assume that the squared Brunt-Väisälä frequency $N_{u}^{2}$ is equal to the mean of the squared total Brunt-Väisälä frequency on the axis of the gravitationally unstable region of the pancake vortex. Similarly, the value of $\Omega$ is taken as the mean value of the angular velocity on the axis of the pancake vortex in the gravitationally unstable region. The stability problem then reduces to the well-known case of a layer heated from below between two parallel plates with free boundary conditions and rotating at constant rate (Chandrasekhar 1961).

We can first note from (4.8) that the most amplified value of $q$ scales like $q_{\max } \sim v^{-1 / 4}\left|N_{u}\right|^{1 / 4} k^{1 / 2}$ when the viscosity $v$ tends to zero and $N_{u}$ is large. Using the fact that $\delta_{z} / \Lambda$ depends only on $\alpha / F_{h}$, we can deduce that the maximum growth rate should vary like $\sigma_{\max } / N \approx \sqrt{-N u^{2}} / N-C / \sqrt{R e F_{h}}$ for large Reynolds number $R e$ and small Froude number $F_{h}$, where $C$ is a constant which depends on $\alpha / F_{h}$ only. This explains the dependence of the growth rate with $R e$ observed in figure $8(a)$. It shows also that for a given value of $\alpha / F_{h}$, the maximum growth rate $\sigma_{\max } / N$ decreases when the Froude number decreases as observed in figure 7(a).

The growth rate predicted by (4.8) with the previous estimates and for $R e=500$, $S c=1$ and $F_{h}=1$ is plotted in figure 11(a) with dashed line. The agreement with the numerical results is satisfactory for $\alpha<0.8 F_{h}$ in view of all of the simplifying assumptions used in the theory. As can be seen in figure 11(b), the numerical frequency scaled by the predicted frequency $m \Omega$ is also around unity. The theoretical growth rate goes to zero for $\alpha=0.87 F_{h}$ (figure 11a). For this value, the Rayleigh and Taylor numbers defined as follows

$$
R a=\frac{-N_{u}^{2} \delta_{z}^{4}}{\nu \kappa}, \quad T a=\frac{4 \Omega^{2} \delta_{z}^{4}}{\nu^{2}},
$$

are $R a=5581$ and $T a=10674$ in agreement with the results of Chandrasekhar (1961). In contrast, the numerical growth rate for $m=1$ goes to zero for $\alpha=1.07 F_{h}$ for which $R a=45$ and $T a=737$. Interestingly, Matthews (1988) has considered the stability of a parabolic density gradient

$$
\frac{\partial \rho_{b}}{\partial z}=B-3 A z^{2}
$$

where $(A, B)$ are positive constants, which corresponds to a gravitationally unstable region within two stable infinitely deep layers. Assuming an unbounded fluid, he found that the critical Rayleigh number for such configuration is much lower $R a=O(100)$. This suggests that the previous assumptions of a uniform density gradient and a bounded fluid along the vertical may be too crude. Thorpe (1994) has computed the maximum growth rate as a function of the Rayleigh number for the density 

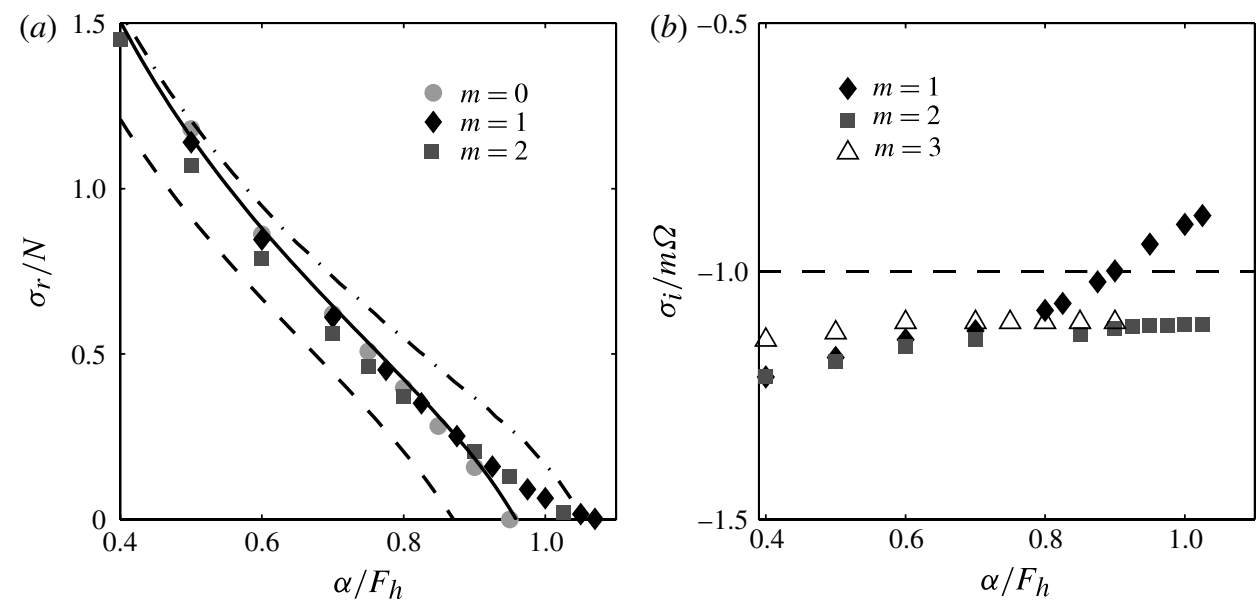

FIgURE 11. (a) Comparison between the theoretical and numerical growth rates as a function of $\alpha / F_{h}$ for $F_{h}=1, R e=500$ and $S c=1$. The solid and dashed lines are the maximum growth rate predicted for the parabolic density gradient (4.11) and for a constant density gradient, respectively. The dash-dotted line is the maximum growth rate obtained for the parabolic density gradient (4.11) without rotation, $T a_{M}=0$, by Thorpe (1994). (b) Numerical frequency scaled by the predicted frequency $m \Omega$ as given in (4.8).

profile (4.11) but only without rotation. We have therefore extended the stability analysis of Matthews (1988) and Thorpe (1994) to take into account a rotation at constant angular velocity $\Omega$.

The vertical gradient of total density on the axis of the pancake vortex in the neighbourhood of the location $z=z_{m} \equiv \Lambda \sqrt{3 / 4}$ where it is maximum approximates to

$$
\frac{\partial \rho_{b}}{\partial z}=-\frac{N^{2} \rho_{0}}{g}\left(1-C+6 \frac{C}{\Lambda^{2}}\left(z-z_{m}\right)^{2}\right)+O\left(z-z_{m}\right)^{3}
$$

where $C=8 F_{h}^{2} \ln 2 \mathrm{e}^{-3 / 2} / \alpha^{2}$. Thus, equation (4.11) will describe well the density gradient (4.12) near $z=z_{m}$ if we set $B=(C-1) N^{2} \rho_{0} / g$ and $A=2 C N^{2} \rho_{0} /\left(g \Lambda^{2}\right)$. The Rayleigh number used by Matthews (1988) and Thorpe (1994) is $R a_{M}=g B d^{4} /\left(\rho_{0} \nu \kappa\right)$ where $d=(B / A)^{1 / 2}$ is a measure of the height of the unstably stratified region. Non-dimensionalizing $z$ with $d$ and writing the vertical velocity in the form $u_{z}=W(z) \mathrm{J}_{m}(q r) \exp (\sigma t+\mathrm{i} m \theta)$, we obtain

$$
\begin{aligned}
& \left(S c S+Q^{2}-\frac{\mathrm{d}^{2}}{\mathrm{~d} z^{2}}\right)\left[\left(S+Q^{2}-\frac{\mathrm{d}^{2}}{\mathrm{~d} z^{2}}\right)^{2}\left(Q^{2}-\frac{\mathrm{d}^{2}}{\mathrm{~d} z^{2}}\right) W-T a_{M} \frac{\mathrm{d}^{2} W}{\mathrm{~d} z^{2}}\right] \\
& =\left(S+Q^{2}-\frac{\mathrm{d}^{2}}{\mathrm{~d} z^{2}}\right)\left[R a_{M}\left(1-3 z^{2}\right) Q^{2} W\right]
\end{aligned}
$$

where $S=d^{2} s / v, Q=q d$ and $T a_{M}=4 \Omega^{2} d^{4} / v^{2}$ is the Taylor number. In the following, we take $\Omega=\Gamma /\left(2 \pi R^{2}\right) \exp \left(-z_{m}^{2} / \Lambda^{2}\right)$, the angular velocity on the axis of the pancake 
vortex at $z=z_{m}$. Taking the Fourier transform of (4.13) yields

$$
\begin{aligned}
& {\left[\left(S c S+Q^{2}+K^{2}\right)\left(S+Q^{2}+K^{2}\right)\left(Q^{2}+K^{2}\right)\right.} \\
& \left.\quad+T a_{M} K^{2} \frac{S c S+Q^{2}+K^{2}}{S+Q^{2}+K^{2}}-R a_{M} Q^{2}\right] \hat{W}=3 R a_{M} Q^{2} \frac{\mathrm{d}^{2} \hat{W}}{\mathrm{~d} K^{2}}
\end{aligned}
$$

where

$$
\hat{W}(K)=\int_{-\infty}^{\infty} \mathrm{e}^{-\mathrm{i} K z} W(z) \mathrm{d} z .
$$

As shown by Matthews (1988), equation (4.14) can be easily solved by a shooting method with the boundary conditions $\hat{W}(K) \rightarrow 0$ at large values of $K$ and $\hat{W}=1$, $\mathrm{d} \hat{W} / \mathrm{d} K=0$ for $K=0$ since $W(z)$ and so $\hat{W}(K)$ should be even for the dominant eigenvalue. The maximum growth rate determined for the set of Rayleigh and Taylor numbers $\left(R a_{M}, T a_{M}\right)$ corresponding to each aspect ratio $\alpha$ for $F_{h}=1, S c=1$ and $R e=500$ is shown by a solid line in figure 11(a). The agreement with the numerical results is very good for $\alpha<0.9 F_{h}$ and much better than the prediction based on (4.8). All of the azimuthal wavenumbers are predicted to have the same growth rate provided that the assumption of a radially unbounded fluid is valid. For $\alpha>0.9 F_{h}$, the predicted growth rate remains in good agreement with the growth rate of the axisymmetric mode $m=0$. In particular, they both vanish around $\alpha=0.95 F_{h}$. In contrast, the azimuthal wavenumbers $m=1,2$ continue to be unstable for larger aspect ratios. The assumption of a radially unbounded fluid is likely to be no longer valid in this range of $\alpha$. In the case of an unstably stratified fluid in an impermeable vertical cylinder, Batchelor \& Nitsche (1993) have found that the azimuthal wavenumbers $m=1$ and $m=2$ become also unstable before the mode $m=0$ when increasing the Rayleigh number. Thus, the radial boundary conditions might favour non-axisymmetric modes.

The maximum growth rate in the absence of rotation (i.e. $\Omega=0$ ) computed by Thorpe (1994) is also shown by a dash-dotted line in figure 11(a). As expected, we see that the rotation is stabilizing.

The growth rates predicted by (4.14) for other values of the horizontal Froude number: $F_{h}=1.5, F_{h}=0.5, F_{h}=0.25(R e=500, S c=1)$ and $F_{h}=0.25, F_{h}=1$ $(R e=1000, S c=1)$ are compared with the numerical results in figure 12(a). Only the growth rate of theazimuthal wavenumber $m=0$ is shown for simplicity. The prediction for the Schmidt number $S c=7$ with $F_{h}=1$ and $R e=500$ is also tested in figure $12(b)$. We can see that the agreement between the theoretical and the numerical growth rate is always very good except for $F_{h}=0.25, R e=500$ where a slight departure is observed. The growth rate predicted for the Schmidt number value $S c=700$ corresponding to salt stratified water is also plotted in figure $12(b)$ even if this value has not been investigated in $\S 3$. The growth rate curves for $S c=700$ and $S c=7$ are in fact very close.

The most amplified value of $q=Q / d, q_{\max }$ for $F_{h}=1, R e=500, S c=1$ is shown by a solid line in figure 13(a). It varies between 9 and 12 in the range of $\alpha$ investigated. The most amplified value of $q$ predicted by (4.8) is similar as shown by a dashed line (figure 13a). The location of the first zero in the radial direction is predicted to be $r_{0}=\mu_{m} / q_{\max }$ where $\mu_{m}$ is the first zero of the Bessel function $\mathrm{J}_{m}$. For $\alpha=0.85 F_{h}$, the most amplified value of the radial wavenumber is $q_{\max }=9.2 / R$. Hence, we have $r_{0} \approx 0.26 R$ for the azimuthal wavenumber $m=0, r_{0} \approx 0.4 R$ for $m=1$ and $r_{0} \approx 0.55 R$ for $m=2$. As seen in figure 5 , this agrees quite well with the radial 

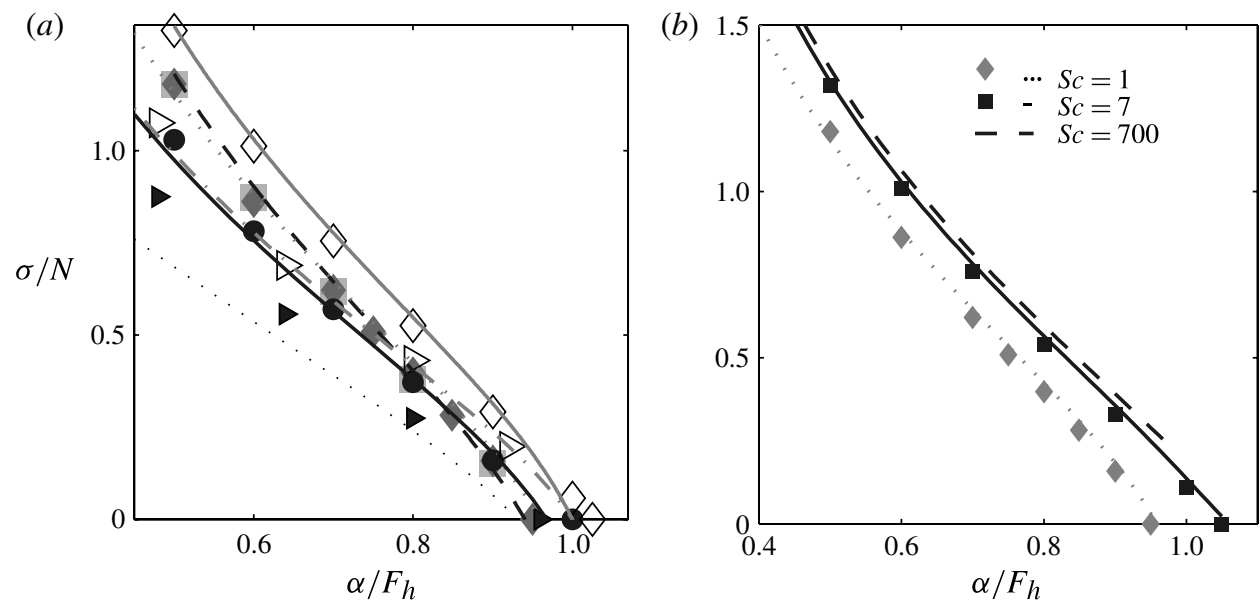

FIGURE 12. Comparison between the theoretical and the numerical growth rates as a function of $\alpha / F_{h}$ for $m=0$. The different lines correspond to the maximum growth rate predicted for the parabolic density gradient (4.11) (a) for different Froude numbers $F_{h}=1.5$

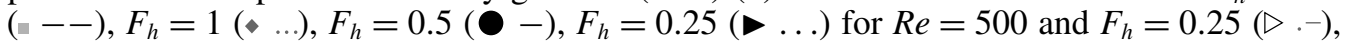
$F_{h}=1.0(\diamond-)$ for $R e=1000$ and a constant $S c=1$ and $(b)$ for $S c=1, S c=7$ and $S c=700$ for $F_{h}=1, R e=500$.
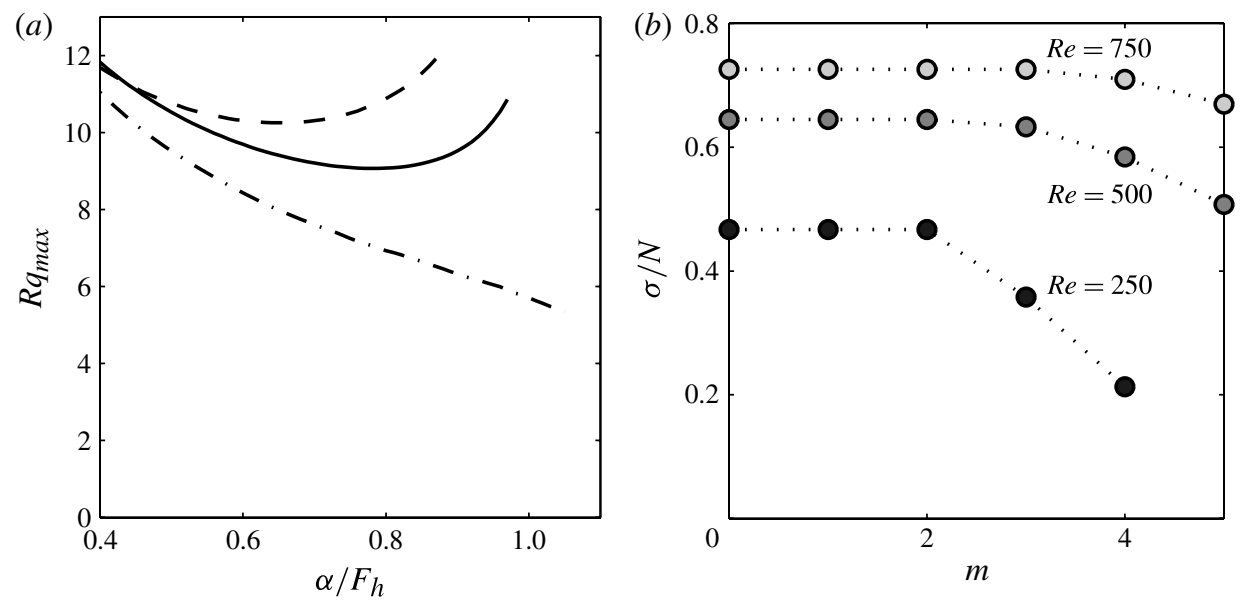

FIgURE 13. (a) Most amplified radial wavenumber $q_{\max }$ as a function of $\alpha / F_{h}$ for $F_{h}=1, R e=500$ and $S c=1$. The solid and dashed lines correspond to the parabolic density gradient (4.11) and a constant density gradient, respectively. The dash-dotted line corresponds to the parabolic density gradient (4.11) without rotation $T a_{M}=0$ (Thorpe 1994). (b) Growth rate predicted for the parabolic density gradient (4.11) as a function of $m$ for $\alpha=0.7 F_{h}$ and for $R e=250,500$ and 750 .

structure of the vertical velocity of the modes obtained in the numerical stability analysis.

However, this implies that the radius of the first node $r_{0}$ will become larger than the radial size $\delta_{r}$ of the unstable region when the azimuthal wavenumber $m$ increases. At this point, it is reasonable to assume that $q$ will be then selected to be approximately $q=\mu_{m} / \delta_{r}$. Figure 13(b) shows the theoretical growth rate as a 
function of the azimuthal wavenumber with $\delta_{r}=0.6$ for $\alpha=0.7 F_{h}$ and the three Reynolds number $R e=[250,500,750]$. The growth rates predicted for the azimuthal wavenumbers $m=0,1$ are in good agreement with the numerical results for all of the Reynolds numbers (figure $8 b$ ). Nevertheless, the growth rate of the azimuthal modes $m \geqslant 3$ decreases with $m$ much slowly than in the numerics (see figure $8 b$ ).

In order to be more quantitative and improve the theory, one would need to take also into account the non-uniformity of the density gradient in the radial direction. Accordingly, it would be also necessary to take into account the variation of the angular velocity $\Omega$ along the vertical. However, such analysis seems much more complicated and not significantly simpler than considering the stability of the full pancake vortex.

\subsection{Comparison with experimental results}

We can try to compare the predicted stability threshold with the previous experiments of Flor \& van Heijst (1996) and Beckers et al. (2001). In these two studies, the velocity profile of the pancake vortex fits well to the profile: $u_{\theta b}=$ $\Omega_{\max } r \exp ^{-r^{2} / R^{2}} \exp ^{-z^{2} / \Lambda^{2}}$. For this Gaussian angular velocity profile, gravitationally unstable regions appear when $\alpha<\alpha_{c}=0.67 F_{h}$, where $F_{h}=\Omega_{\max } / N$. The radius of the pancake vortices in the experiments of Flor \& van Heijst (1996) is around $4 \mathrm{~cm}$ while their thickness is approximately $\Lambda \sim 3 \sqrt{2} \mathrm{~cm}$ giving an aspect ratio around unity. They reported that they were unstable if $F \geqslant 0.1$ with a Froude number defined as $F=V_{\max } /\left(N R_{\max }\right)$ where $V_{\max }$ is the maximum azimuthal velocity and $R_{\max }$ the corresponding radius. The relation with the present definition for the Froude number is $F=0.61 F_{h}$ so that the threshold $F \geqslant 0.1$ corresponds to $F_{h} \geqslant 0.16$. This critical Froude number is therefore much smaller than the threshold $F_{h}>1.5$ for the gravitational instability for $\alpha \approx 1$, but the instability reported by Flor \& van Heijst (1996) is of different nature: it is a radial shear instability due to the inflection point present in the radial vorticity profile. Beckers et al. (2001) have also measured both the radial and vertical velocity profiles of one pancake vortex and they estimated that the Froude number is $F=0.3$ and the aspect ratio $\alpha=0.3 \sqrt{2}$. Their definition of the Froude number is related to that used herein by $F=2 F_{h} * \sqrt{\pi} \alpha$. Therefore, we have $\alpha / F_{h}=2.1$ which is well above the stability threshold $\alpha_{c} / F_{h}=0.67$.

\section{Generalization to arbitrary velocity profiles}

The results presented so far have been obtained for the specific case of a Lamb-Oseen vortex with a Gaussian distribution in the vertical direction. For this case, a gravitational unstable region appear when decreasing the aspect ratio, whereas the necessary condition for a vertical shear instability $0<\min (R i)<1 / 4$ is never satisfied. The aim of this section is to determine to what extent this result depends on the vortex profile. To this end, we consider general velocity profiles of the form

$$
\tilde{u}_{\theta b}=h(\tilde{r}) f(\tilde{z})
$$

where $\tilde{r}=r / R, \tilde{z}=z / \Lambda$ and $\tilde{u}_{\theta b}=u_{\theta b} / \Gamma / 2 \pi R$. For this general velocity profile, the Richardson number reads

$$
R i=\frac{\frac{\alpha^{2}}{F_{h}^{2}}-2\left(f f^{\prime}\right)^{\prime} \mathscr{I}_{G}}{f^{\prime 2} h^{2}},
$$


where $\mathscr{I}_{G}=\int_{\tilde{r}}^{\infty} h^{2}(\mathscr{R}) / \mathscr{R} \mathrm{d} \mathscr{R}$ and the prime denotes differentiation with respect to the variable $\tilde{z}$. The necessary condition for the shear instability $0<\min (R i)<1 / 4$ will be satisfied if the vertical gradient of total density is everywhere negative, i.e.

$$
\frac{\alpha^{2}}{F_{h}^{2}}>2 \max \left[\left(f f^{\prime}\right)^{\prime} \mathscr{I}_{G}\right]
$$

and if

$$
\frac{\alpha^{2}}{F_{h}^{2}}<2\left(f f^{\prime}\right)^{\prime}\left(\tilde{z}_{s}\right) \mathscr{I}_{G}\left(\tilde{r}_{s}\right)+\frac{1}{4} h^{2}\left(\tilde{r}_{s}\right) f^{\prime 2}\left(\tilde{z}_{s}\right)
$$

where $\tilde{r}=\tilde{r}_{s}, \tilde{z}=\tilde{z}_{s}$ is the point where the Richardson number is minimum. Combining (5.3) and (5.4) shows that we must have

$$
2 \max \left[\left(f f^{\prime}\right)^{\prime} \mathscr{I}_{G}\right]<2\left(f f^{\prime}\right)^{\prime}\left(\tilde{z}_{s}\right) \mathscr{I}_{G}\left(\tilde{r}_{s}\right)+\frac{1}{4} h^{2}\left(\tilde{r}_{s}\right) f^{\prime 2}\left(\tilde{z}_{s}\right) .
$$

Since $\mathscr{I}_{G}$ is monotonically decreasing with $\tilde{r}$, it is maximum at $\tilde{r}=0$. Let us denote $\tilde{z}_{m}$ the vertical level where $f^{\prime}$ is maximum. Since $f^{\prime \prime}\left(\tilde{z}_{m}\right)=0$, we have $f^{\prime 2}\left(\tilde{z}_{s}\right) \leqslant f^{\prime 2}\left(\tilde{z}_{m}\right)=f^{\prime 2}\left(\tilde{z}_{m}\right)+f\left(\tilde{z}_{m}\right) f^{\prime \prime}\left(\tilde{z}_{m}\right) \leqslant \max \left[\left(f f^{\prime}\right)^{\prime}\right]$. Evidently, we have also $\left(f f^{\prime}\right)^{\prime}\left(\tilde{z}_{s}\right) \leqslant \max \left[\left(f f^{\prime}\right)^{\prime}\right]$. Therefore, a necessary condition ensuring that (5.5) can be fulfilled is

$$
\mathscr{I}_{G}(0)<\mathscr{I}_{G}\left(\tilde{r}_{s}\right)+\frac{1}{8} h^{2}\left(\tilde{r}_{s}\right)
$$

This condition is interesting since it involves only the radial profile $h(\tilde{r})$ of azimuthal velocity. Since $h(0)=0$ for smooth vortices, equation (5.6) requires that the function $F(\tilde{r})=\mathscr{I}_{G}(\tilde{r})+(1 / 8) h^{2}(\tilde{r})$ has a maximum value larger than $F(0)$. This first requires that the derivative $F^{\prime}(\tilde{r})=-h^{2} / \tilde{r}+h h^{\prime} / 4$ is positive for some finite radius $\tilde{r}$ (primes denote now differentiation with respect to $r$ ). In terms of angular velocity $\Omega(\tilde{r})=h(\tilde{r}) / \tilde{r}$, the latter condition reads

$$
F^{\prime}(\tilde{r})=\frac{\Omega \tilde{r}}{4}\left[-3 \Omega+\tilde{r} \Omega^{\prime}\right]>0,
$$

or, equivalently,

$$
F^{\prime}(\tilde{r})=\frac{\Omega \tilde{r}}{4}[-5 \Omega+\zeta]>0,
$$

where $\zeta=\tilde{r} \Omega^{\prime}+2 \Omega$ is the vertical vorticity. We can see easily that the condition (5.7) is never satisfied for classical vortices since the angular velocity is generally decreasing monotonically with $\tilde{r}$, i.e. $\Omega^{\prime}<0$. In fact, (5.7)-(5.8) are very restrictive since (5.8) shows that it is necessary to have some radius where the vertical vorticity is five times larger than the angular velocity.

This condition is stringent because the vertical shear of azimuthal velocity implies an important anomaly of density through the thermal wind relation (2.5). When decreasing the aspect ratio, the vertical gradient of total density becomes then positive in the vortex core before that the vertical shear is sufficiently strong for the Kelvin-Helmholtz instability to develop. Since this effect is intimately related to the balance (2.1) between the radial gradient of pressure and the centrifugal acceleration, we may expect that (5.7) is satisfied for flows where curvature effects are smaller than for classical vortices. For example, for a hollow vortex with the radial profile $h(\tilde{r})=\tilde{r} \mathrm{e}^{-\left(\tilde{r}-R_{c}\right)^{2}}$, the condition (5.7) is satisfied when the characteristic radius $R_{c}$ is large: $R_{c}>\sqrt{6}$. The condition (5.7) is only a necessary condition and in practice, the 
condition (5.5) can be satisfied only when $R_{c}>2.95$ for the vertical profile $f(\tilde{z})=\mathrm{e}^{-\tilde{z}^{2}}$. We note that for such a large $R_{c}$, this vortex profile could be approximated, locally, as a jet since the curvature is small.

The conditions (5.6) and (5.7) apply only to azimuthal velocity profiles that are separable in the vertical and radial directions. For general velocity profiles of the form $\tilde{u}_{\theta b}=f(\tilde{r}, \tilde{z})$, we did not succeed in deriving a general necessary condition. The conditions for the shear and gravitational instabilities have thus to be computed for each vortex profile. For example, in the case of the vertical vorticity profile

$$
\omega_{z b}(\tilde{r}, \tilde{z})=2\left[1-\tanh \left(\tilde{r}^{2}+\tilde{z}^{2}\right)\right],
$$

the threshold for the gravitational instability is $\alpha_{c}=1.24 F_{h}$ while the Richardson criterion (4.3) is never satisfied: $\min (R i)$ is larger than 1.1 for $\alpha>1.24 F_{h}$ and then becomes abruptly negative for $\alpha<1.24 F_{h}$. Therefore, the same conclusion as for the Lamb-Oseen vortex applies to this particular profile.

\section{Criterion for the centrifugal instability}

In a stratified, inviscid and incompressible fluid, the Rayleigh criterion for the centrifugal instability is modified to (Solberg 1933; Elliassen \& Kleinschmidt 1957)

$$
\Phi=\left.\frac{1}{r^{3}} \frac{\partial\left(r u_{\theta b}\right)^{2}}{\partial r}\right|_{\rho_{b}}<0
$$

somewhere in the flow. The only difference with the Rayleigh criterion in homogeneous fluids is that the radial derivative should be taken along isopycnal surfaces. This criterion can be easily derived heuristically by considering the variation of total energy resulting from the exchange of two fluid particles. Ooyama (1966) has shown rigourously that (6.1) is a necessary and sufficient condition for the instability of axisymmetric disturbances. Note that such inertial instability is generally called 'symmetric instability' in the meteorological literature.

The quantity $\Phi$ can also be expressed in terms of the potential vorticity $\Pi=\vec{\omega} \cdot \overrightarrow{\nabla \rho}$ (Hoskins 1974). Indeed, we have

$$
\Phi=\left.\frac{1}{r^{3}} \frac{\partial}{\partial r}\left(r u_{\theta b}\right)^{2}\right|_{z}-\frac{1}{r^{3}} \frac{\partial}{\partial z}\left(r u_{\theta b}\right)^{2} \mid \frac{\frac{\partial \rho_{b}}{\partial r}}{\frac{\partial \rho_{b}}{\partial z}}=\frac{2}{r} u_{\theta b} \frac{\Pi}{\frac{\partial \rho_{b}}{\partial z}} .
$$

The latter relation shows that the instability condition (6.1) is equivalent to $\Pi>0$ for stably stratified fluids $\partial \rho_{b} / \partial z \leqslant 0$ if $u_{\theta b} \geqslant 0$ as in (2.1). The contours of $\Phi$ for the velocity profile (2.1) and density profile (2.6) are shown in figure 14 for $\alpha=1.12$. This aspect ratio is just above the limit for instability $\alpha=1.112$. We see that $\Phi$ is slightly negative (grey regions in figure 14) in the outer regions $r_{\min }>1.5 R$ because the potential vorticity of the basic vortex (2.1) and (2.6) is not zero but slightly positive outside the vortex core. However, the values of $\Phi$ are very small so that it is expected to be only marginally unstable and probably explain why such instability has not been observed.

\section{Conclusions}

We have investigated the stability of a pancake vortex with a Gaussian vorticity profile in a stratified fluid as a function of its aspect ratio $\alpha$, the horizontal Froude 


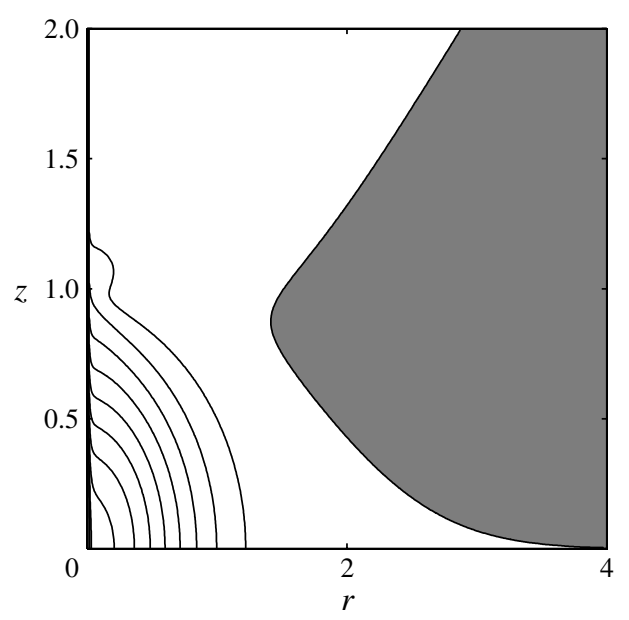

FIGURE 14. Contours of $\Phi / \Phi_{\max }$ given by (6.2) in the $(r, z)$-plane for the profile (2.1) and $\alpha=1.12$ which implies a stable stratification everywhere. In the white regions $\Phi>0$ while in the grey regions $\Phi$ is slightly negative. The contour interval is 0.5 .

number $F_{h}$, the Reynolds number Re and the Schmidt number $S c$. The vorticity of such vortex is single-signed suggesting that it should never be unstable to a 'radial' two-dimensional shear instability. When decreasing the aspect ratio, we have shown that the first and dominant instability is a gravitational instability related to the density anomaly due to the thermal wind balance. The predicted critical aspect ratio $\alpha_{c}=1.11 F_{h}$ for the gravitational instability is in very good agreement with the critical value obtained in the numerical stability analysis $\alpha_{c} \sim 1.1 F_{h}$ for sufficiently large Reynolds number.

In order to explain the properties of the gravitational instability, we have extended the stability analysis of Matthews (1988) and Thorpe (1994) of a viscous diffusive fluid with a parabolic vertical density gradient to include a rotation at constant angular velocity. Such flow resembles the unstably stratified region embedded within the pancake vortex. The comparison between the theoretical and numerical results reveal a good agreement. Both the numerical results and the theoretical predictions show that decreasing the aspect ratio destabilizes a larger number of azimuthal wavenumbers $m$, but the modes $m=0,1,2$ remain dominant. A similar behaviour is observed when increasing the Reynolds number: the band of unstable azimuthal wavenumber $m$ is widened and the growth rate of each azimuthal mode increases.

Finally, the dominance of the gravitational instability over the vertical shear instability has been shown to hold for a general class of pancake vortices with angular velocity of the form $\tilde{\Omega}(r, z)=\Omega(r) f(z)$ provided that $r \partial \Omega / \partial r<3 \Omega$ everywhere. This condition does not involve the vertical profile of angular velocity $f(z)$. It shows that the vertical shear instability should never occur on classical vortices since $\Omega$ generally decreases monotonically with the radius. However, hollow pancake vortices in which curvature effects are weaker than for vortices might be unstable to a shear instability instead of the gravitational instability if they are sufficiently hollow and if the vertical shear is sufficiently strong.

Even if the basic flow investigated here is very simple, we may envision some general consequences for stratified turbulence. The present study suggests that the 
gravitational instability should play a role as important as the shear instability in the dynamic of small vertical length scales. However, in contrast to the shear instability that generates horizontal billows of the order of the buoyancy length scale (Waite 2011; Augier \& Billant 2011), the gravitational instability destabilizes a wide range of horizontal length scales whose small-scale cut-off is controlled by diffusive effects. Therefore, it may transfer some energy directly to dissipative scales. Hence, it would be interesting to further investigate its role and its effect in the energy cascade in stratified turbulence.

\section{Acknowledgements}

This work was supported by the National Research Agency (ANR-06-BLAN-0363) and by a Marie Curie Intra European Fellowship within the seventh Framework Programme (PIEF-GA-2009-234782). Special thanks go to D. Guy for technical support.

\section{REFERENCES}

Abramowitz, M. \& Stegun, I. A. 1970 Handbook of Mathematical Functions, (ed. M. Abramowitz \& I.A. Stegun), Dover Publications.

Augier, P. \& Billant, P. 2011 Onset of secondary instabilities on the zigzag instability in stratified fluids. J. Fluid Mech. 682, 120-131.

BAtChelor, G. K. \& Nitsche, J. M. 1993 Instability of stratified fluid in a vertical cylinder. J. Fluid Mech. 252, 419-448.

Beckers, M., Clercx, H. J. H., van Heijst, G. J. F. \& Verzicco, R. 2003 Evolution and instability of monopolar vortices in a stratified fluid. Phys. Fluids 15, 1033-1045.

Beckers, M., Verzicco, R., Clercx, H. J. H. \& VAN Heisst, G. J. F. 2001 Dynamics of pancake-like vortices in a stratified fluid: experiments, model and numerical simulations. J. Fluid Mech. 433, 1-27.

BILlant, P. 2010 Zigzag instability of vortex pairs in stratified and rotating fluids. Part 1: general stability equations. J. Fluid Mech. 660, 354-395.

Billant, P. \& Chomaz, J.-M. 2000a Experimental evidence for a new instability of a vertical columnar vortex pair in a strongly stratified fluid. J. Fluid Mech. 418, 167-188.

Billant, P., Deloncle, A., Chomaz, J.-M. \& Otheguy, P. 2010 Zigzag instability of vortex pairs in stratified and rotating fluids. Part 2: analytical and numerical analyses. J. Fluid Mech. 660, 396-429.

Bonnier, M., EifF, O. \& Bonneton, P. 2000 On the density structure of far-wake vortices in a stratified fluid. Dyn. Atmos. Oceans 31, 117-137.

Brethouwer, G., Billant, P., Lindborg, E. \& Chomaz, J.-M. 2007 Scaling analysis and simulation of strongly stratified turbulent flows. J. Fluid Mech. 585, 343-368.

Chandrasekhar, S. 1961 Hydrodynamic and Hydromagnetic Stability, pp. 87-105. Oxford University Press/Dover Publications.

Delbende, I., Chomaz, J.-M. \& Huerre, P. 1998 Absolute/convective instabilities in the Batchelor vortex. J. Fluid Mech. 355, 229-254.

Deloncle, A., Billant, P. \& Chomaz, J.-M. 2008 Nonlinear evolution of the zigzag instability in stratified fluids: a shortcut on the route to dissipation. J. Fluid Mech. 599, 229-239.

Elliassen, E. \& KLEINSCHMIDT, E. 1957 Stability of a compressible baroclinic vortex in a gravity field. Dyn. Meteor. Geophys. II, Handbuch der Physik 48, 66-71.

Flor, J.-B. \& VAN HeIJst, G. J. F 1996 Stable and unstable monopolar vortices in a stratified fluid. J. Fluid Mech. 311, 257-287.

FUNG, Y. T. 1986 Non-axisymmetric waves of a stratified vertical vortex. J. Appl. Mech. 29 (2), 368-371.

FUnG, Y. T. 1992 Richardson criteria for stratified vortex motions under gravity. Phys. Fluids 59, 445 . 
Godoy-Diana, R. \& Chomaz, J.-M. 2003 Effect of the Schmidt number on the diffusion of axisymmetric pancake vortices in a stratified fluid. Phys. Fluids 15, 1058-1064.

Hoskins, B. J. 1974 The role of potential vorticity in symmetric stability and instability. Quart. J. Roy. Meteor. Soc. 100, 480-482.

Lin, S. J. \& Pierrehumbert, R. T. 1987 Comment on Richardson criteria for stratified vortex motions under gravity. Phys. Fluids 30 (4), 1231-1232.

Matthews, P. C. 1988 A model for the onset of penetrative convection. J. Fluid Mech. 188, 571-583.

OOYAMA, K. 1966 On the stability of the baroclinic circular vortex: a sufficient criterion for instability. J. Atmos. Sci. 23, 43-53.

Otheguy, P., Chomaz, J.-M. \& Billant, P. 2006 Elliptic and zigzag instabilities on co-rotating vertical vortices in a stratified fluid. J. Fluid Mech. 553, 253-272.

Riley, J. J. \& DEBRUYNKops, S. M. 2003 Dynamics of turbulence strongly influenced by buoyancy. Phys. Fluids 15, 2047-2059.

Riley, J. J. \& LELONG, M.-P. 2000 Fluid motion in the presence of strong stable stratification. Annu. Rev. Fluid Mech. 32, 613-657.

SOlBerG, H. 1933 Le mouvement d'inertie de l'atmosphere stable et son role dans la theorie des cyclones. In Meteor. Assoc. U.G.G.I., (ed. Lisbon), vol. 1, pp. 66-82. Dupont.

Thorpe, S. A. 1994 The stability of statically unstable layers. J. Fluid Mech. 260, 315-331.

Waite, M. L. 2011 Stratified turbulence at the buoyancy scale. Phys. Fluids 23 (6), 066602.

Waite, M. L. \& SMolarkiewicz, P. K. 2008 Instability and breakdown of a vertical vortex pair in a strongly stratified fluid. J. Fluid Mech. 606, 239-273. 